\title{
32. MAGNETOSTRATIGRAPHY OF SITES 782, 783, 784, AND 786, IZU-BONIN OUTER FOREARC, WESTERN PACIFIC ${ }^{1}$
}

\author{
J. R. Ali, ${ }^{2}$ R. B. Haston, ${ }^{3}$ and L. B. Stokking ${ }^{4}$
}

\begin{abstract}
During Leg 125, scientists drilled Sites $782,783,784$, and 786 across a transect of the Izu-Bonin forearc near $31^{\circ} \mathrm{N}$. Magnetostratigraphy for whole-core and discrete specimens has been integrated with biostratigraphic data and correlated to the geomagnetic polarity time scale. These correlations are good back to the middle Miocene at Sites 783, 784, and 786 and to the late Oligocene at Site 782, but become more tentative in older sediments because of poor recovery and complex magnetizations.
\end{abstract}

\section{INTRODUCTION}

Ocean Drilling Program (ODP) Sites 782, 783, 784, and 786 form a transect close to $31 \% \mathrm{~N}$ across the outer part of the Izu-Bonin forearc. Paleomagnetic, biostratigraphic, and sedimentological data provide scientists an opportunity to investigate the evolution of this forearcterrain, where the Pacific plate has been subducting beneath the Philippine Sea plate since the Eocene. The paleomagnetic record obtained from the forearc sequences recovered at these sites is generally good from the middle/late Miocene to the present. Earlier than that, a combination of low sediment accumulation, sedimentary hiatuses, unstable magnetizations, and poorer biostratigraphic data (because of calcite dissolution) makes correlation with the standard geomagnetic polarity time scale (GPTS) of Berggren et al. (1985) less reliable. However, several welldefined magnetochron boundaries that have been correlated with this scale permit one to calculate sedimentation rates in the Izu-Bonin forearc. Future work with these data will help develop a depositional history for the area that can be used to unravel the enigmatic tectonic history of the Izu-Bonin forearc.

\section{LITHOLOGIC UNITS}

Hole 782A (Fig. 1) was drilled on the eastern margin of the Izu-Bonin forearc basin, about halfway between the active volcanic arc and the trench. Two lithologic units were identified in this hole. Unit I ( $0-409.2 \mathrm{~m}$ below seafloor, or mbsf) comprises a sedimentary succession from Pleistocene to late Eocene in age and is divided into three subunits (Subunit IA, 0-153.6 mbsf; Subunit IB, 153.6-337.0 mbsf; and Subunit IC, $337.0-409.2 \mathrm{mbsf}$ ). This unit consists primarily of nannofossil-rich marls and some chalks, together with abundant volcanic debris. Recovery through Unit I averaged just under $70 \%$. Two unconformities have been identified in the sediments: one between upper Oligocene and lower Miocene sediments and another between upper Eocene and lower Oligocene sediments. Unit II (409.2-476.8 mbsf), recovered as gravel-sized chips, comprises intermediate-acid volcanic rocks that form basement.

Site 783 (Fig. 1) is located on the northern mid-flank portion of the Torishima forearc serpentinite seamount, on the inner wall of the Izu-Bonin trench. Two lithologic units were identified in the single hole drilled at this site. Unit I (0-120.0 mbsf) comprises a mid-Pleis-

\footnotetext{
${ }^{1}$ Fryer, P., Pearce, J. A., Stokking, L. B., et al., 1992, Proc. ODP, Sci. Results, 125: College Station, TX (Ocean Drilling Program).

${ }^{2}$ Department of Oceanography, University of Southampton, SO9 $5 \mathrm{NH}$, United Kingdom.

${ }^{3}$ Department of Geological Sciences, University of California, Santa Barbara, CA 93106, U.S.A. (Present address: AMOCO Production Company, 501 West Lake Park Boulevard, P.O. Box 3092, Houston, TX 77253, U.S.A.)

${ }^{4}$ Ocean Drilling Program, Texas A\&M University, 1000 Discovery Drive, College Station, TX 77845-9547, U.S.A.
}

tocene to lower Pliocene, or older, sequence of glass-rich clay and claystone. Unit II (120.0-158.6 mbsf) is a phacoidal sheared serpentine (Fryer, Pearce, Stokking, et al., 1990) that contains clasts of harzburgite, but was not sampled for this study.

Hole 784A (Fig. 1) is located on the western flank of the same seamount. Two lithologic units were identified in this hole. Unit I is subdivided into Subunits IA (0-126.4 mbsf), IB (126.4-302.7 mbsf), and IC (302.7-321.1 mbsf). It consists primarily of clays and claystone together with a significant vitric component. Subunit IC also contains a notable amount of clay and silt-sized serpentine. Unit II (321.1-425.3 mbsf) is a phacoidal sheared serpentine microbreccia.

Site 786 (Fig. 1) is located in the center of the Izu-Bonin forearc basin, about $200 \mathrm{~km}$ east of the active volcano Myojin Sho. Three sedimentary units were identified in Hole 786A. Unit I (0-83.46 mbsf) consists of lower Pleistocene to middle Miocene nannofossil marls and clays. Unit II (83.46-103.25 mbsf) comprises a sequence of upper Oligocene to middle Eocene nannofossil marls and nannofossil-rich clays with abundant volcaniclastic debris. Unit III (103.25-124.90 mbsf) is a series of volcaniclastic breccias. Unit IV (Hole 786A: 124.9-166.5 mbsf; Hole 786B: 162.5-826.6 mbsf) is a volcanic sequence that forms basement.

\section{METHODS}

Paleomagnetic measurements of sediments from the Izu-Bonin forearc were performed using (1) the shipboard three-axis whole-core 2G-cryogenic (WCC) magnetometer and (2) the discrete-specimen fluxgate (DSF) magnetometer (manufactured by Molspin) in the Southampton University paleomagnetic laboratory. The WCC magnetometer was used to measure paleomagnetism at $10-\mathrm{cm}$ intervals along the length of all archive-half cores, whereas the DSF was used for discrete specimens contained in standard $7-\mathrm{cm}^{3}$ ODP paleomagnetic sample cubes. Typically, one discrete specimen was taken from each 1.5-m section of core. Alternating field (AF) demagnetization was used to remove low-stability (low-coercivity) components of magnetization. The WCC system has a three-axis AF demagnetizer mounted in line with the magnetometer, and the archive-half cores were demagnetized to a maximum field of $10 \mathrm{mT}$. The discrete specimens were demagnetized using a Molspin rotating specimen system to maximum fields of 40 to $50 \mathrm{mT}$. The cores are unorientedpolarity is assessed on the basis of inclination only.

At Site 782, a total of 179 specimens was taken from Unit I for this study. The gravel-sized chips of Unit II were too broken-up for paleomagnetic work. Twenty-five specimens were taken from the upper $66.01 \mathrm{~m}$ of Unit I at Site 783. In the lower half of the unit, the core recovery was low, and the material retrieved was too disturbed for paleomagnetic work. No samples were taken for this investigation from the sheared phacoidal serpentine of Unit II. A total of 126 paleomagnetic specimens was taken from Unit I at Site 784; no 

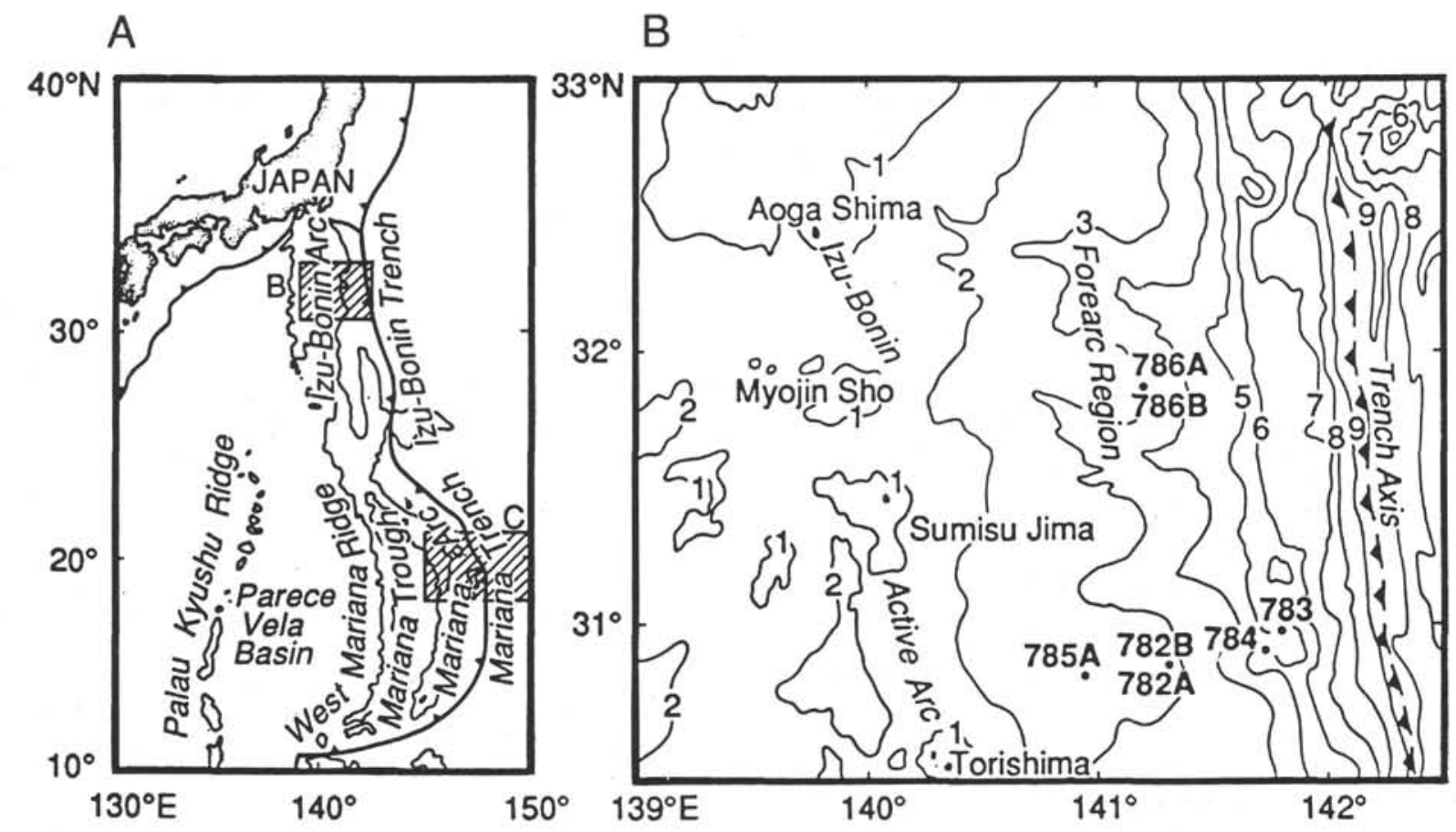

Figure 1. Location of Sites 782, 783, 784, and 786 in the Izu-Bonin forearc.

samples were analyzed from Unit II, a sheared phacoidal serpentine microbreccia. Thirty discrete samples were taken from Unit I at Site 786 for this study. Although paleomagnetic specimens were taken from Unit IV at Site 786, a volcanic basement sequence, none of the data is presented in this paper.

\section{AF DEMAGNETIZATION AND MAGNETIC STABILITY}

All archive-half cores, except those showing noticeable drilling disturbance, were processed using the WCC. Measurements of the natural remanent magnetization (NRM) and the remanence remaining after demagnetization to $10 \mathrm{mT}$ were conducted on the cores; the intensity typically decreased to about $20 \%$ to $40 \%$ of NRM after demagnetization at $10 \mathrm{mT}$. Changes in both the remanence declination and inclination of up to a few tens of degrees are typical (see Appendix A).

As nearly all of the discrete specimens had NRM intensities of $>20 \mathrm{~mA} / \mathrm{m}$, these were processed using the DSF (noise level $=<0.05$ $\mathrm{mA} / \mathrm{m}$ ), together with a Molspin tumbler, for demagnetization to 40 to $50 \mathrm{mT}$. Unfortunately, a small number of the specimens from Holes $782 \mathrm{~A}(7 \%)$ and $784 \mathrm{~A}(4 \%)$ and a significant proportion from Hole $786 \mathrm{~A}(41 \%)$ proved too unstable to measure beyond values of about $15 \mathrm{mT}$, and these data should be ignored. Note that all these specimens are from intervals where the sedimentation rate is a few meters per million years (Fryer, Pearce, Stokking, et al., 1990).

Typical examples of demagnetization data from the discrete specimens are shown in Figure 2. In all cases, small to moderate changes in the direction of remanent magnetism occur during demagnetization of up to 5 to $10 \mathrm{mT}$, but thereafter the trajectories are generally directed toward the origin.

\section{MAGNETIC POLARITIES AND STRATIGRAPHIC CORRELATIONS}

The magnetic polarity history for each hole is based on both the WCC data (Appendix A) and DSF data (Appendix B). These data have been used to define a polarity log (Figs. 3 through 6 ) for each of the holes, and the DSF data have been used to verify the much higher resolution WCC magnetostratigraphy. The WCC records include a number of magnetozones (up to a few tens of meters) that contain short (a few tens of centimeters) intervals of opposite polarity. Discrete-specimen data from a number of these levels confirm these short-term polarity inversions within the WCC record.

Biostratigraphic control is provided by calcareous nannofossils (Ciampo, this volume; $\mathrm{Xu}$ and Wise, this volume) and diatoms (Stabell, this volume). The sequences of magnetic polarity zones identified in each of the holes are matched with the GPTS of Berggren et al. (1985).

\section{Hole 782A}

The magnetostratigraphic record for Hole 782A(Fig. 3) is reliable between 0 and $322 \mathrm{mbsf}$, where there is close agreement between the WCC and DSF data. Below this level, the DSF data are in many cases of opposite polarity to the equivalent level in the WCC record, indicating that $10-\mathrm{mT}$ demagnetization was insufficient to remove the effects of secondary magnetizations. In the lower part of this hole, the WCC record is dominated by thinner magnetozones. However, the biostratigraphic data at these levels indicate sedimentation rates that average about 1 to $3 \mathrm{~m} / \mathrm{m}$.y., and these therefore may represent a condensed magnetostratigraphic record. As insufficient DSF data exist from these levels to confirm the WCC results, one should not place too much emphasis on the WCC data.

The start of the normal polarity Brunhes Chron is positioned at 24.8 mbsf. The reverse polarity Matuyama Chron begins at 66.20 mbsf and includes records of the Jaramillo (29.1-34.6 mbsf) and Olduvai (47.8-53.5 mbsf) normal polarity subchrons. Incomplete recovery and poor biostratigraphic control prevented us from locating the normal polarity Gauss Chron precisely, but normal polarity intervals corresponding to this chron have been recorded in Cores 125$782 \mathrm{~A}-8 \mathrm{H}$ to $125-782 \mathrm{~A}-12 \mathrm{X}(66.8-115.0 \mathrm{mbsf})$. All these cores have been assigned to nannofossil Zone $\mathrm{CN} 12$. Portions of the reversed 

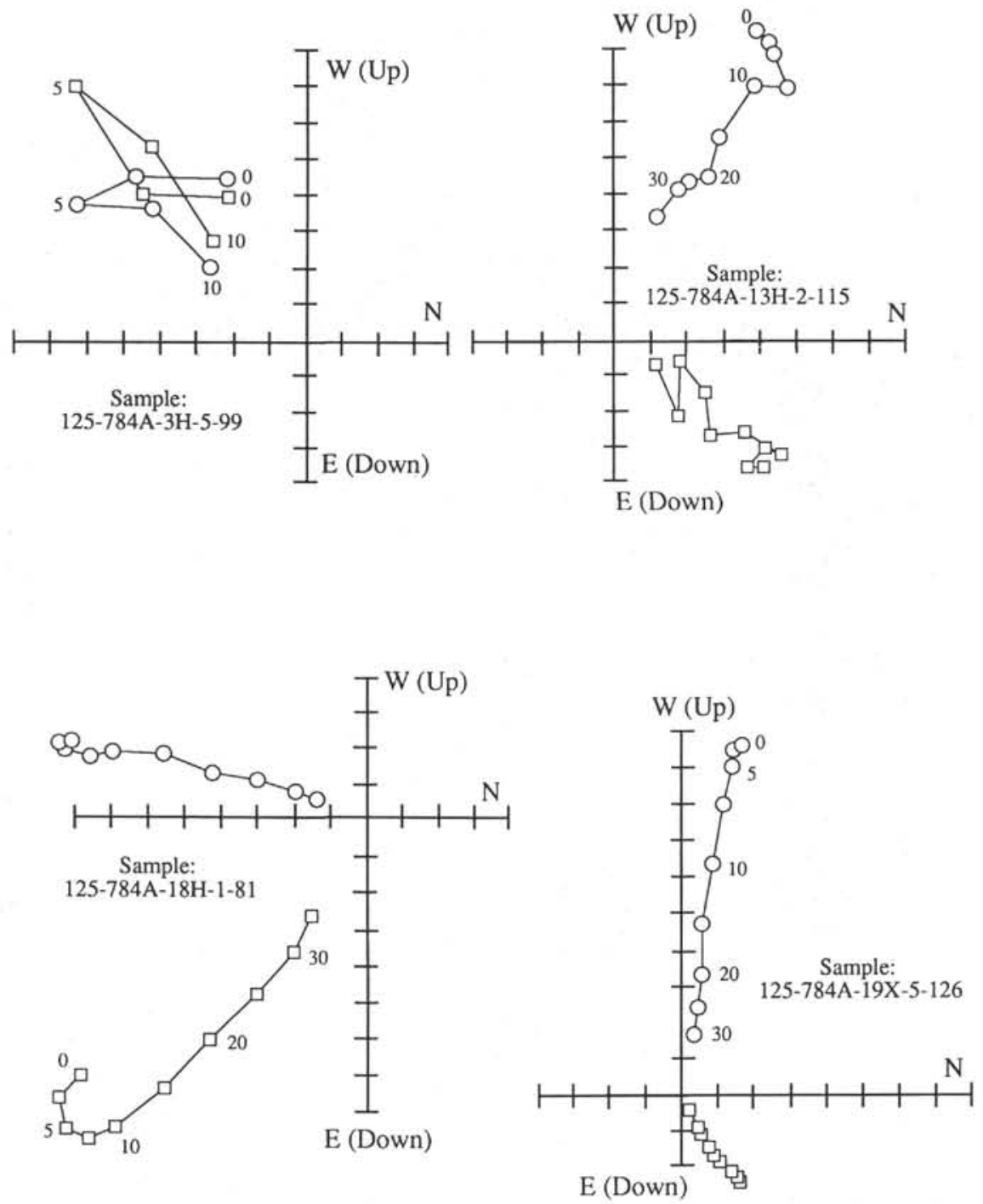

Figure 2. Vector end-point diagrams showing the AF demagnetization behavior of typical discrete specimens from Hole 784A. Demagnetization data are for $5,10,15$ or 20,30 , and $40 \mathrm{mT}$. "N," "E," "S," and "W" refer to an arbitrary reference azimuth because the specimens are from unoriented cores. Intensities have been normalized to natural remanent magnetization values. Circles represent projection onto the vertical plane; squares represent projection onto the horizontal plane.

polarity Gilbert Chron were identified within Cores $125-782 \mathrm{~A}-13 \mathrm{X}$ to $125-782 \mathrm{~A}-16 \mathrm{X}$ (117.7 to $160.2 \mathrm{mbsf}$ ) and include partial records of the Cochiti, Nunivak, and Thvera normal polarity subchrons.

Between 160.2 and $245.5 \mathrm{mbsf}$, the cores have been assigned to nannofossil Zone CN9 and the lower half of Zone 10A. Assuming a constant sedimentation rate (about $25 \mathrm{~m} / \mathrm{m}$.y.) throughout this part of the succession, the hole probably contains partial records of Chron 5 through to Chron 9.

Cores 125-782A-26X and 125-782A-27X (240.3-259.6 mbsf) exhibit predominantly normal polarity. The nannofossil Zones CN6 (248.6-253.7 mbsf) and CN7 (245.5-248.6 mbsf) are succeeded by Zone CN9 (the base of which is defined at $245.5 \mathrm{mbsf}$ ). Chron C5 is associated with Zones $\mathrm{CN} 6$ and $\mathrm{CN} 7$, and a correlation of the interval from 245.5 to $253.7 \mathrm{mbsf}$ with this chron is proposed. The base of Zone CN9 is within the same block of normal polarity; thus the normal polarity sediments above 245.7 mbsf must correlate with parts of Chron 9. The hiatus inferred by the absence of nannofossil Zone CN8 from the succession is supported by the apparently discontinuous paleomagnetic record and by the lack of reversed polarity sediments that would correlate with the early part of Chron 9 and with Chron 10.

Cores 125-782A-28X to $125-782 \mathrm{~A}-34 \mathrm{X}$ (259.6-322.5 mbsf) contain nannofossil Zone CN5(a/b), which spans 3.6 m.y. One can correlate the normal polarity intervals within this sequence to Chrons C5A (261.8-267.25 mbsf), C5AAN (278.8-282.1 mbsf), C5ABN (289.35-282.1 mbsf), C5ACN (297.55-300.1 mbsf), and C5AD (303.5-308.7 mbsf).

Correlation below this level is more tentative. Chrons $\mathrm{C} 8, \mathrm{C} 9$, and $\mathrm{C} 10$ (within the late Oligocene nannofossil Zone CP19) may occupy parts of the interval between 332.1 and $351.0 \mathrm{mbsf}$, below which correlation is impossible.

\section{Hole 783A}

Only the sediments from the upper half of Unit I in Hole 783A were suitable for paleomagnetic studies. The polarity record (Fig. 4) contains two normal polarity magnetozones (identified in Cores 
Site 782

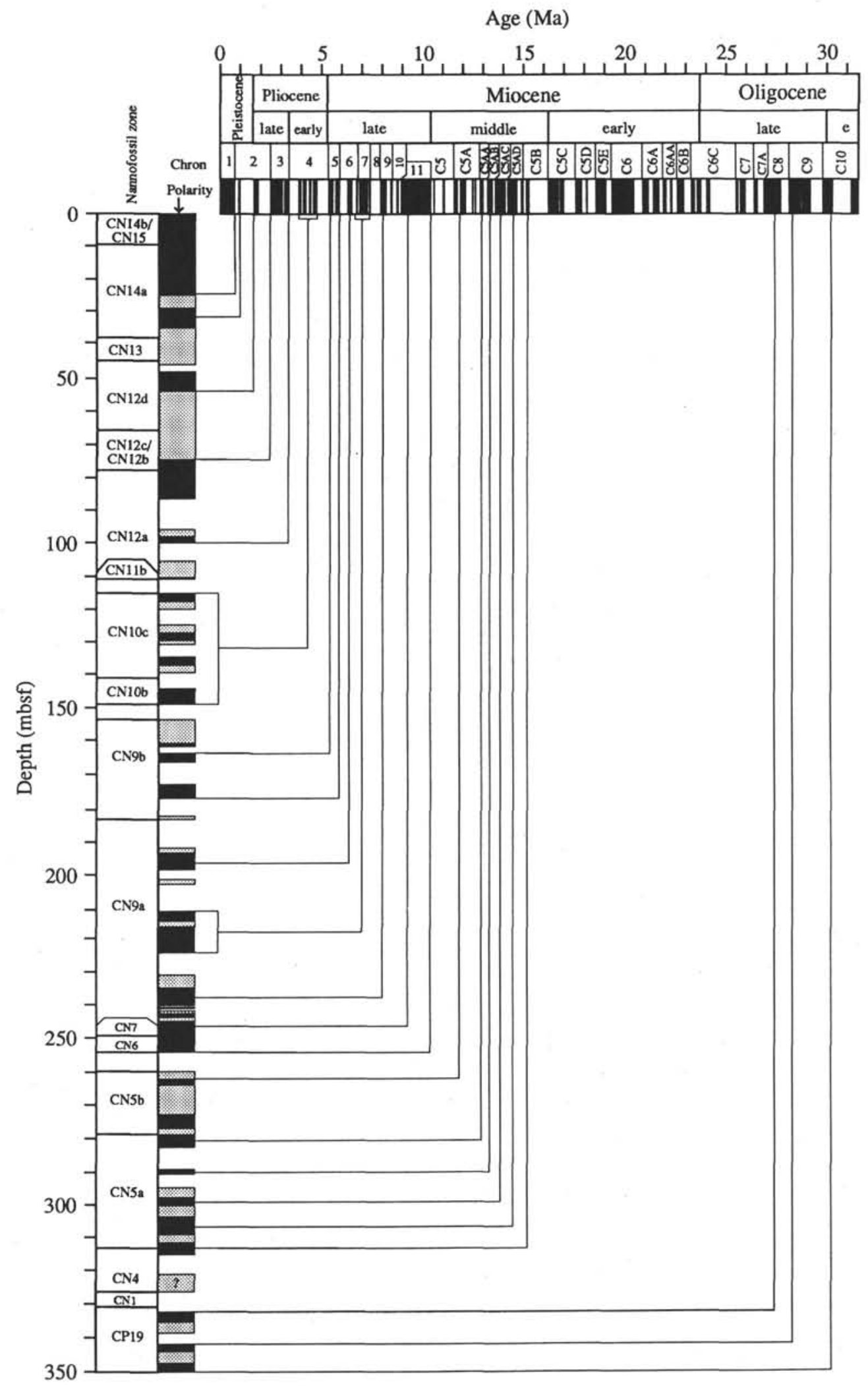

Figure 3. Correlation of the magnetic polarity record for Hole 782A (to $350 \mathrm{mbsf}$ ) with the GPTS of Berggren et al. (1985). Black indicates normal polarity, the stippled pattern represents reversed polarity, and blank areas indicate regions of no recovery or no data. 
Site 783

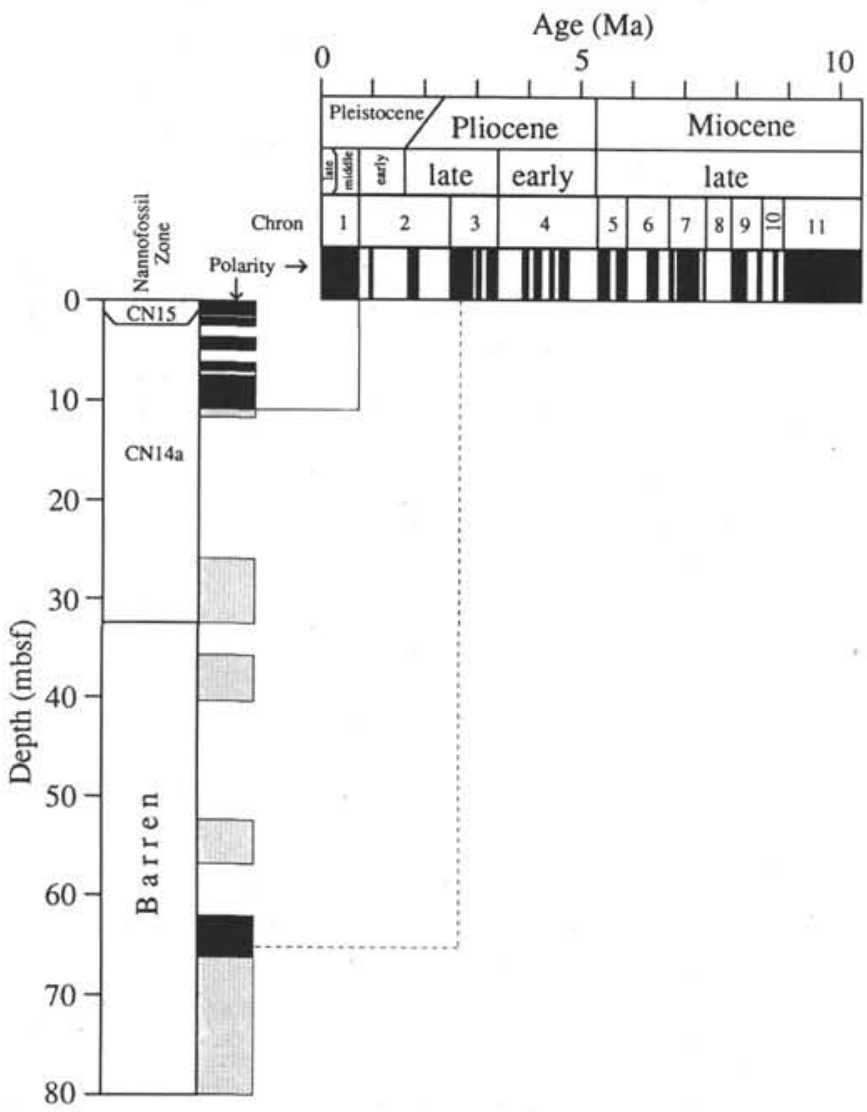

Figure 4. Correlation of the magnetic polarity record for Hole 783A (to $80 \mathrm{mbsf}$ ) with the GPTS of Berggren et al. (1985) via the scale of Barron (1985). Black indicates normal polarity, the stippled pattern represents reversed polarity, and blank areas indicate regions of no recovery or no data. The broken line indicates that the correlation is tentative.

125-783A-1R, 0-9.7 mbsf, and 125-783A-8R, 62.1-71.8 mbsf) separated by an interval of reversed polarity. Unfortunately, no polarity transitions have been identified within any of the cores.

Biostratigraphic data for Hole 783A are based primarily on diatoms; because of calcite dissolution only two calcareous nannofossil datums have been identified. Correlations with the GPTS are via the scale proposed by Barron (1985). Core 125-783-1R (0-9.7 mbsf) contains the diatom Zones Nitzschia reinholdii and Pseudoeunotia doliolus, which indicates an early/middle Pleistocene age. The normal polarity magnetization within this core is a record of the Brunhes Chron. Cores 125-783A-2R (9.7-16.4 mbsf), 125-783A-4R (26.0$35.7 \mathrm{mbsf}), 125-783 \mathrm{~A}-5 \mathrm{R}$ (35.7-42.7 mbsf), and 125-783A-7R (52.4-62.1 mbsf) contain faunal assemblages that correlate with the nannofossil CN14A Zone and diatom Rhizosolenia praebergonii Zone; thus, these cores are early Pliocene to middle Pleistocene in age. The reversed polarity magnetization within these four cores records the Matuyama Chron. Unfortunately, core recovery at these levels averaged $35 \%$, and none of the normal polarity subchrons within the Matuyama Chron have been identified. The normal polarity Gauss Chron is represented in Core 125-783A-8R (62.1-71.8 mbsf).

\section{Hole 784A}

Although the sedimentary sequence in Hole 784A extends to 321.1 mbsf, below about 190 mbsf (Core 127-784A-21R) structural com-
Site 784

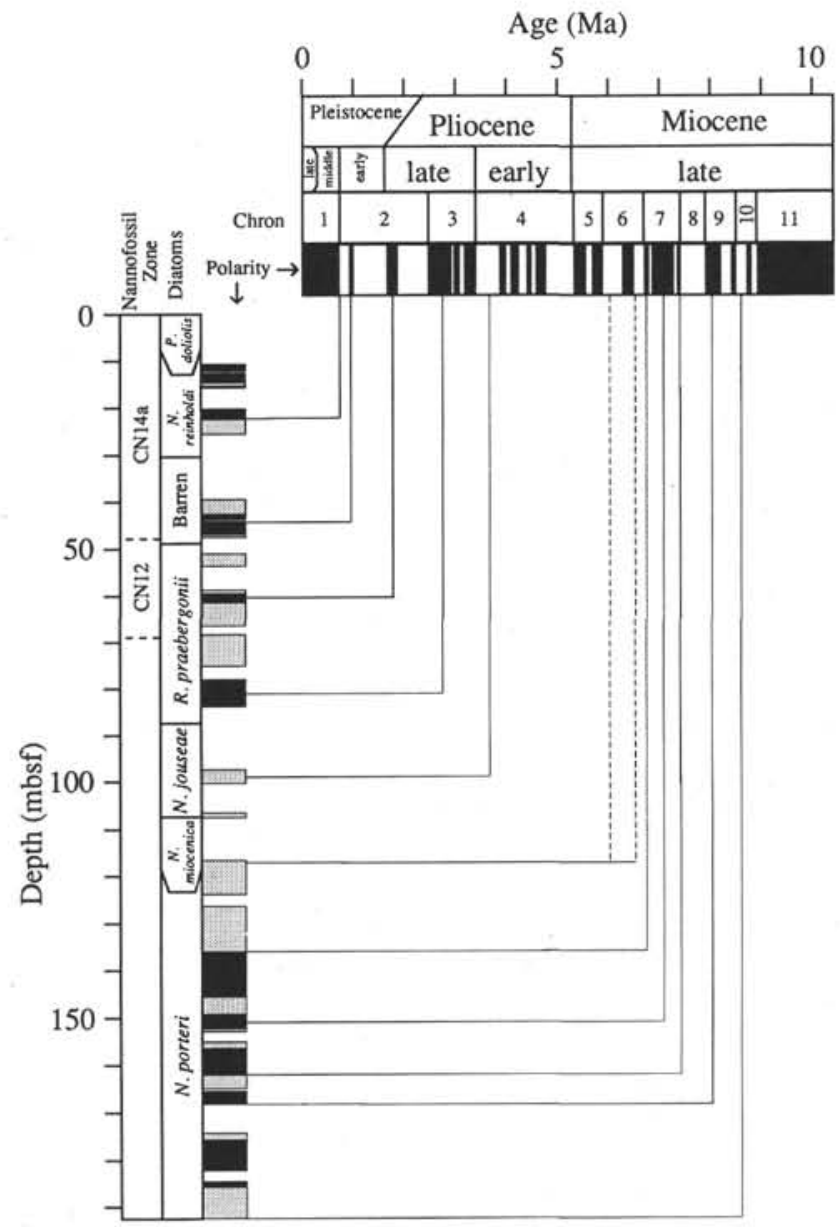

Figure 5. Correlation of the magnetic polarity record for Hole 784A (to $190 \mathrm{mbsf}$ ) with the GPTS of Berggren et al. (1985) via the scale of Barron (1985). Black indicates normal polarity, the stippled pattern represents reversed polarity, and blank areas indicate regions of no recovery or no data. Correlations indicated by broken lines are more tentative than those indicated by solid lines.

plications that include shear zones, normal and reverse faults, and bedding dips ranging from $35^{\circ}$ to $70^{\circ}$ (Fryer, Pearce, Stokking, et al., 1990 , pp. 285-289) make correlation with the GPTS unreliable. Biostratigraphic information from the recovered sediments is limited to a sparse diatom assemblage and two calcareous nannofossil events; core recovery averaged only $56.6 \%$. We present tentative correlations from the upper part of the hole (Fig. 5).

The start of the normal polarity Brunhes Chron was recorded at 22.4 mbsf (within Core 125-784A-4R, 20.4-29.9 mbsf). The Jaramillo and Olduvai normal polarity subchrons, within the reversed polarity Matuyama Chron, are positioned between 42.75 and 47.0 mbsf and 59.5 and 61.6 mbsf, respectively. A partial record of the normal polarity Gauss Chron was identified between 78.1 and 83.9 mbsf; this probably represents the youngest normal polarity interval assigned to this chron. A small portion of the reversed polarity Gilbert Chron probably is represented between 97.4 to $100.2 \mathrm{mbsf}$, as this interval was assigned to the Nitzschia jouseae diatom zone.

Sediments in Cores 125-784A-14R and 125-784A-15R (116.7 to $136.0 \mathrm{mbsf}$ ) are assigned to the $N$. jouseae and Nitzschia porteri diatom zones. Thus, intervals of reversed polarity within these cores 
Site 786

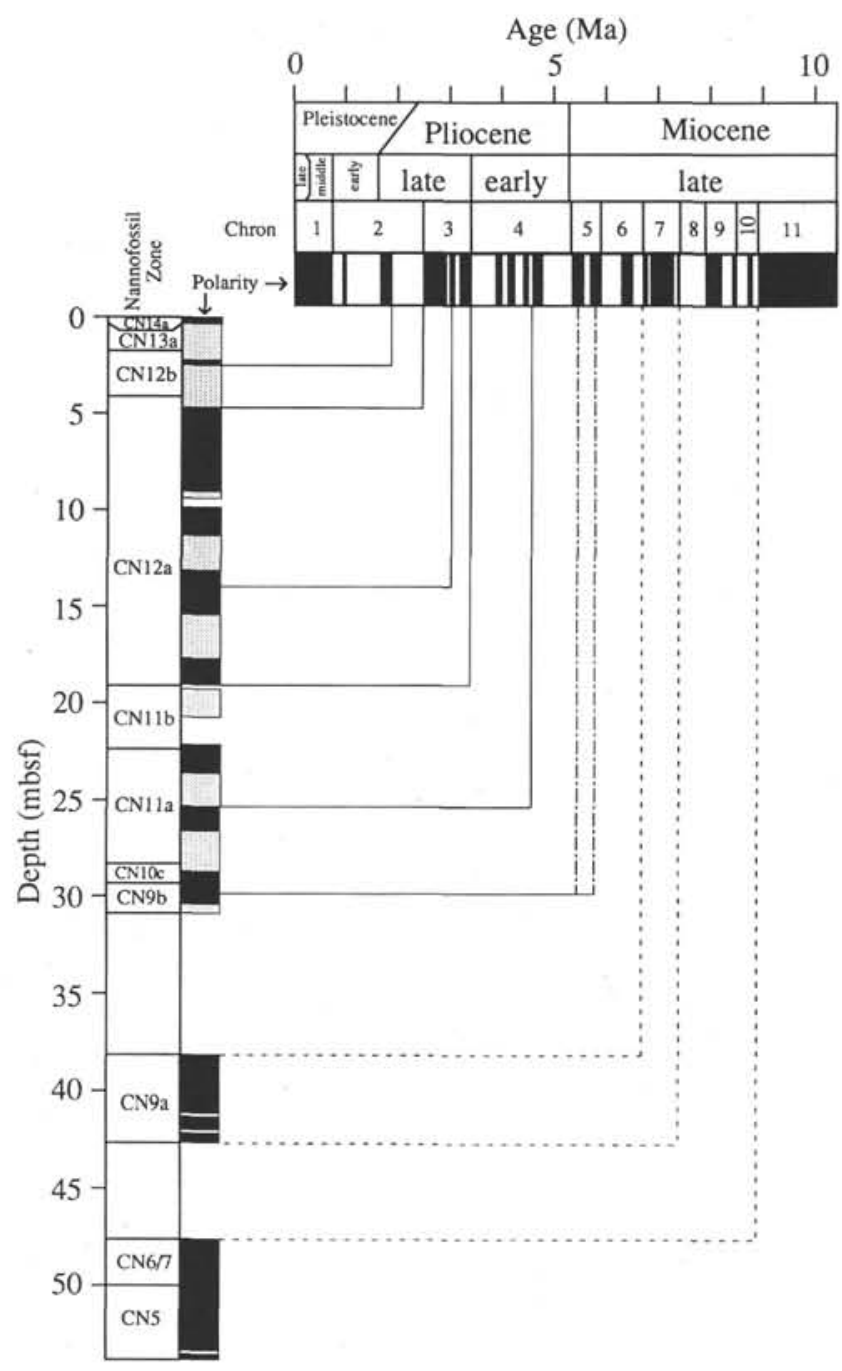

Figure 6. Correlation of the magnetic polarity record for Hole 786A (to 55 mbsf) with the GPTS of Berggren et al. (1985). Black indicates normal polarity, the stippled pattern represents reversed polarity, and blank areas indicate regions of no recovery or no data. Correlations indicated by broken lines are more tentative than those indicated by solid lines. The dashed-anddotted lines indicate two possible correlations.

probably represent portions of Chron 5 and the later part of Chron 6 . No record was found of the Thalassiosira convexa Zone that Barron (1985) observed between these two zones.

Cores $125-784 \mathrm{~A}-16 \mathrm{R}$ to $125-784 \mathrm{~A}-20 \mathrm{R}$ (136.00-182.1 mbsf) were assigned to the $N$. porteri $Z$ Zone. The polarity sequence recorded by these cores may represent portions of Chrons 6 (lower part) and 7 . The identification of the Coscinodiscus yabei Zone, identified in Core 125-784A-21R (184.4-194.0 mbsf), reinforces this correlation.

\section{Hole 786A}

Interpretation of the WCC and DSF data (Fig. 6) has been restricted to the upper six cores $(53.95 \mathrm{mbsf})$. From this level to Core 125-786A-11X (96.0-105.7 mbsf), the DSF specimens exhibit complex magnetic behavior; obtaining reliable data from these levels proved impossible. Cores $125-786 \mathrm{~A}-1 \mathrm{H}$ to $125-786 \mathrm{~A}-6 \mathrm{X}(0-57.1$ mbsf) range in age from early/middle Pleistocene (Zone CN14) to middle Miocene (CN5 at $53.95 \mathrm{mbsf}$ ).

The reversed polarity Matuyama Chron has been interpreted as beginning at $4.65 \mathrm{mbsf}$; the interval between 2.2 and 2.5 mbsf may record the Olduvai normal polarity subchron. The interval from 4.65 to $19.15 \mathrm{mbsf}$ was assigned to the nannofossil Zone CN12a, and thus may represent the Gauss Chron.

The polarity sequence observed between 28.7 and $30.4 \mathrm{mbsf}$ suggests that this interval may contain a partial record of Chron 5 . The interval from 38.2 to 42.65 mbsf contains nannofossils from Zone $\mathrm{CN} 9 \mathrm{a}$, which correlates with Chron 7. Chron C5N was partially recorded between 47.6 and 53.95 mbsf, based on the presence of nannofossils from the upper part of Zone CN5 and from Zone CN6/7.

\section{CONCLUSIONS}

During Leg 125, Sites 782, 783, 784, and 786 were drilled across a transect of the Izu-Bonin forearc near $31^{\circ} \mathrm{N}$ that yielded sedimentary sequences suitable for magnetostratigraphic analysis. Paleomagnetic data from whole-core and discrete samples were integrated with biostratigraphic data. Although the biostratigraphic data are poor in many places, reasonable correlations to the geomagnetic polarity time scale back to the middle Miocene were performed on cores from Holes 783A, 784A, and 786A. The data from Hole 782A enabled us to extend these correlations back to the late Oligocene.

Although some correlations were attempted in the older sequences, the poor recovery, complex magnetizations, and sparse biostratigraphic control make these correlations much more tentative.

\section{REFERENCES}

Barron, J. A., 1985. Miocene to Holocene planktonic diatoms. In Bolli, H. M., Saunders, J. B., and Perch-Nielsen, K. (Eds.), Plankton Stratigraphy: Cambridge (Cambridge Univ. Press), 763-809.

Berggren, W. A., Kent, D. V., Flynn, J. J., and Van Couvering, J. A., 1985. Cenozoic geochronology. Geol. Soc. Am. Bull., 96:1407-1418.

Fryer, P., Pearce, J. A., Stokking, L. B., et al., 1990. Proc. ODP, Init. Repts., 125: College Station, TX (Ocean Drilling Program).

Date of initial receipt: 1 October 1990

Date of acceptance: 6 May 1991

Ms 125B-155 


\section{APPENDIX A}

WCC Magnetometer Polarity Data for Leg 125

The interpretations are based on the changes in remanence between the natural remanent magnetization direction and direction after demagnetization to $10 \mathrm{mT}$ of the archive cores. Many of the defined polarity intervals are based on the "trend" toward a particular polarity state $(\mathrm{N}$, normal; R, reversed; ?, indeterminate). From these data it is possible to make a polarity evaluation based on the relative shift in direction toward a particular polarity state. Generally, this shift is greatest for levels with a reversed polarity depositional remanence because the antiparallel normal polarity viscous remanent magnetization (VRM) is removed during the early stages of demagnetization ( 5 to $10 \mathrm{mT}$ ). Normal polarity intervals characteristically exhibit smaller changes in direction (less than $10^{\circ}$, as the VRM is aligned subparallel to the detrital remanent magnetization), but up to a $50 \%$ decrease in intensity. The position of several thin $(10-20 \mathrm{~cm})$ "anomalous" levels is indicated in the "Comments" column.

\begin{tabular}{cccc}
\hline & \multicolumn{2}{c}{ Depth (mbsf) } & \\
\cline { 3 - 3 } Core & Polarity & Upper Lower & Comments \\
\hline
\end{tabular}

$125-782 \mathrm{~A}-$

\begin{tabular}{|c|c|c|c|c|}
\hline \multirow[t]{3}{*}{ IH } & $\mathrm{N}$ & 0.0 & 7.70 & \\
\hline & $\mathbf{R}$ & & 8.10 & Section 4 not measured at $10 \mathrm{mT}$ \\
\hline & $\mathrm{N}$ & & 9.80 & $\begin{array}{l}\text { Two low-inclination swings at } 13.00 \\
\text { and } 13.90 \mathrm{mbsf}\end{array}$ \\
\hline \multirow[t]{2}{*}{$2 \mathrm{H}$} & $\mathrm{N}$ & 9.80 & 17.25 & \\
\hline & $?$ & & 18.80 & No section \\
\hline \multirow[t]{2}{*}{$3 \mathrm{H}$} & $\mathrm{N}$ & 19.30 & 24.80 & $\begin{array}{l}\text { Notable declination/inclination } \\
\text { swing at } 25.70 \mathrm{mbsf}\end{array}$ \\
\hline & $\mathrm{R}$ & & 28.80 & \\
\hline \multirow[t]{2}{*}{$4 \mathrm{H}$} & $\mathrm{N}$ & 29.10 & 34.60 & \\
\hline & $?$ & & 35.20 & \\
\hline $5 \mathrm{H}$ & & & & Mix-up with core depth record \\
\hline \multirow[t]{2}{*}{$6 \mathrm{H}$} & $\mathrm{N}$ & 47.80 & 53.50 & $\mathrm{R}$ at $51.25 \mathrm{mbsf}$ \\
\hline & $\mathbf{R}$ & & 57.20 & \\
\hline \multirow[t]{16}{*}{$7 \mathrm{H}$} & $?$ & 57.30 & 57.80 & \\
\hline & N & & 59.25 & \\
\hline & N? & & 60.65 & \\
\hline & $\mathrm{N}$ & & 61.00 & \\
\hline & $\mathbf{R}$ & & 62.75 & \\
\hline & $\mathrm{N}$ & & 62.90 & \\
\hline & $\mathbf{R}$ & & 63.15 & \\
\hline & $\mathrm{N}$ & & 63.65 & \\
\hline & $\mathrm{R}$ & & 64.25 & \\
\hline & $\mathrm{N}$ & & 64.70 & \\
\hline & $\mathbf{R}$ & & 65.00 & \\
\hline & $\mathrm{N}$ & & 65.20 & \\
\hline & $\mathrm{R}$ & & 65.50 & \\
\hline & $\mathrm{N}$ & & 65.85 & \\
\hline & $\mathrm{R}$ & & 66.20 & \\
\hline & $\mathrm{N}$ & & 66.80 & \\
\hline \multirow[t]{10}{*}{$8 \mathrm{H}$} & $\mathrm{R}$ & 66.80 & 67.35 & \\
\hline & $\mathrm{N}$ & & 68.10 & \\
\hline & $\mathbf{R}$ & & 69.10 & \\
\hline & $\mathrm{N}$ & & 69.50 & \\
\hline & $\mathrm{R}$ & & 70.10 & \\
\hline & $\mathrm{N}$ & & 70.40 & \\
\hline & $\mathbf{R}$ & & 70.50 & \\
\hline & $\mathrm{N}$ & & 70.95 & \\
\hline & $\mathrm{R}$ & & 74.40 & \\
\hline & $\mathrm{N}$ & & 76.30 & \\
\hline \multirow[t]{5}{*}{$9 \mathrm{H}$} & $\mathrm{R}$ & 76.30 & 77.60 & \\
\hline & $?$ & & 79.20 & \\
\hline & $\mathrm{N}$ & & 85.20 & \\
\hline & $?$ & & 86.00 & \\
\hline & $N$ & & 86.30 & \\
\hline $10 \mathrm{X}$ & & & & No core \\
\hline \multirow[t]{2}{*}{$11 X$} & $\mathrm{R}$ & 95.70 & 97.90 & \\
\hline & $\mathrm{N}$ & & 99.60 & \\
\hline \multirow[t]{2}{*}{$12 \mathrm{X}$} & $\mathbf{R}$ & 105.30 & 110.05 & \\
\hline & $\mathrm{N}$ & & 110.30 & \\
\hline \multirow[t]{4}{*}{$13 \mathrm{X}$} & $\mathrm{R}$ & 115.00 & 115.45 & \\
\hline & $\mathrm{N}$ & & 115.90 & \\
\hline & $?$ & & 116.20 & \\
\hline & $\mathrm{N}$ & & 117.70 & \\
\hline
\end{tabular}

APPENDIX A (continued).

\begin{tabular}{|c|c|c|c|c|}
\hline \multirow[b]{2}{*}{ Core } & \multirow[b]{2}{*}{ Polarity } & \multicolumn{2}{|c|}{ Depth (mbsf) } & \multirow[b]{2}{*}{ Comments } \\
\hline & & Upper & Lower & \\
\hline & $\mathrm{R}$ & & 120.00 & \\
\hline \multirow[t]{3}{*}{$14 \mathrm{X}$} & $\mathrm{R}$ & 124.60 & 126.00 & \\
\hline & $\mathrm{N}$ & & 129.30 & \\
\hline & $\mathrm{R}$ & & 130.60 & \\
\hline \multirow[t]{2}{*}{$15 \mathrm{X}$} & $\mathrm{N}$ & 134.30 & 136.70 & \\
\hline & $\mathrm{R}$ & & 139.00 & \\
\hline $16 \mathrm{X}$ & $\mathrm{N}$ & 143.90 & 148.05 & Good swings toward $\mathrm{N}$ at $10 \mathrm{mT}$ \\
\hline \multirow[t]{2}{*}{$17 \mathrm{X}$} & $\mathbf{R}$ & 153.60 & 160.20 & \\
\hline & $\mathrm{N}$ & & 161.20 & \\
\hline $18 \mathrm{X}$ & $\mathrm{N}$ & 163.20 & 166.00 & \\
\hline $19 \mathrm{X}$ & $\mathrm{N}$ & 172.90 & 177.90 & \\
\hline $20 X$ & $\mathrm{R}$ & 182.50 & 183.50 & \\
\hline \multirow[t]{2}{*}{$21 X$} & $\mathrm{R}$ & 192.10 & 193.60 & \\
\hline & $\mathrm{N}$ & & 198.90 & \\
\hline $22 \mathrm{X}$ & $\mathrm{R}$ & 201.80 & 203.30 & \\
\hline $23 \mathrm{X}$ & $\mathrm{N}$ & 211.50 & 214.40 & \\
\hline & $\mathrm{R}$ & & 216.00 & \\
\hline $24 \mathrm{X}$ & $\mathrm{N}$ & & 224.60 & \\
\hline $25 \mathrm{X}$ & $\mathrm{R}$ & 230.70 & 235.40 & \\
\hline & $\mathrm{N}$ & & 236.10 & \\
\hline & $\mathrm{R}$ & & 236.35 & \\
\hline & $\mathrm{N}$ & & 239.70 & \\
\hline & $?$ & & 240.05 & \\
\hline $26 x$ & $\mathrm{R}$ & 240.30 & 240.50 & \\
\hline & ? & & 241.30 & \\
\hline & $\mathbf{R}$ & & 242.00 & \\
\hline & $\mathrm{N}$ & & 243.30 & \\
\hline & $\mathrm{R}$ & & 244.10 & \\
\hline & $\mathrm{N}$ & & 244.30 & \\
\hline & $\mathrm{R}$ & & 244.50 & \\
\hline $27 X$ & $\mathrm{~N}$ & & 253.70 & \\
\hline $28 \mathrm{X}$ & $\mathrm{R}$ & 259.60 & 261.80 & \\
\hline & $\mathrm{N}$ & & 263.75 & \\
\hline & $\mathrm{R}$ & & 264.40 & \\
\hline & $\mathrm{N}$ & & 264.75 & \\
\hline & $\mathrm{R}$ & & 265.30 & \\
\hline & $\mathrm{N}$ & & 266.10 & \\
\hline & $\mathrm{R}$ & & 267.00 & \\
\hline & $\mathrm{N}$ & & 267.25 & \\
\hline & $\mathrm{R}$ & & 268.60 & \\
\hline $29 X$ & $\mathrm{R}$ & 269.20 & 272.40 & \\
\hline & $\mathrm{N}$ & & 276.70 & \\
\hline & $\mathrm{R}$ & & 278.50 & \\
\hline $30 x$ & $\mathrm{~N}$ & 278.80 & 281.30 & \\
\hline & $\mathrm{R}$ & & 281.45 & \\
\hline & $\mathrm{N}$ & & 281.55 & \\
\hline & $\mathrm{R}$ & & 281.85 & \\
\hline & $\mathrm{N}$ & & 282.10 & \\
\hline $31 x$ & $\mathrm{R}$ & 289.00 & 289.35 & \\
\hline & $\mathrm{N}$ & & 290.50 & \\
\hline & ? & & 291.40 & \\
\hline $32 X$ & ? & 294.00 & 297.55 & No WCC magnetometer data \\
\hline & $\mathrm{N}$ & & 300.10 & \\
\hline $33 \mathrm{X}$ & $\mathrm{N}$ & 303.50 & 308.70 & \\
\hline & $\mathrm{R}$ & & 311.10 & \\
\hline & $\mathrm{N}$ & & 313.00 & \\
\hline $34 X$ & $\mathrm{~N}$ & 313.10 & 315.00 & \\
\hline $35 \mathrm{X}$ & $\mathrm{N}$ & 322.50 & 323.35 & \\
\hline & $\mathrm{R}$ & & 324.15 & \\
\hline & $\mathrm{N}$ & & 324.40 & \\
\hline & $\mathrm{R}$ & & 325.10 & \\
\hline & $\mathrm{N}$ & & 327.30 & \\
\hline & $\mathrm{R}$ & & 327.90 & \\
\hline & $\mathrm{N}$ & & 328.10 & \\
\hline & $\mathrm{R}$ & & 328.90 & \\
\hline & $\mathrm{N}$ & & 331.50 & \\
\hline & $\mathrm{R}$ & & 332.00 & \\
\hline $36 \mathrm{X}$ & $\mathrm{N}$ & 332.10 & 335.50 & \\
\hline & $\mathrm{R}$ & & 335.90 & \\
\hline & $\mathrm{N}$ & & 336.40 & \\
\hline & $\mathrm{R}$ & & 336.85 & \\
\hline & $\mathrm{N}$ & & 337.15 & \\
\hline & $\mathrm{R}$ & & 338.70 & $\begin{array}{l}\text { Plus two } \mathrm{N} \text { trends at } 337.75 \text { and } \\
338.2 \text { mbsf }\end{array}$ \\
\hline & $\mathrm{N}$ & & 338.85 & \\
\hline & $\mathrm{R}$ & & 339.20 & $\mathrm{~N}$ at $339.4 \mathrm{mbsf}$ \\
\hline
\end{tabular}


APPENDIX A (continued).

\begin{tabular}{|c|c|c|c|c|}
\hline \multirow[b]{2}{*}{ Core } & \multirow{2}{*}{ Polarity } & \multicolumn{2}{|c|}{ Depth (mbsf) } & \multirow[b]{2}{*}{ Comments } \\
\hline & & Upper & Lower & \\
\hline \multirow[t]{4}{*}{$37 \mathrm{X}$} & $\mathrm{N}$ & 341.60 & 345.50 & $\mathrm{R}$ at $344.8 \mathrm{mbsf}$ \\
\hline & $\mathrm{R}$ & & 347.35 & $\mathrm{~N}$ at 346.3 and $346.7 \mathrm{mbsf}$ \\
\hline & $\mathrm{N}$ & & 349.80 & $\mathrm{R}$ at $349.35 \mathrm{mbsf}$ \\
\hline & $\mathrm{R}$ & & 351.00 & $\mathrm{~N}$ at 350.35 and $351.10 \mathrm{mbsf}$ \\
\hline $38 \mathrm{X}$ & ? & & & Core too short \\
\hline $39 \mathrm{X}$ & $?$ & & & $\mathrm{~N}-\mathrm{R}-\mathrm{N}$ at about $363 \mathrm{mbsf}$ \\
\hline \multirow[t]{6}{*}{$40 \mathrm{X}$} & $\mathrm{N}$ & 370.50 & 374.20 & \\
\hline & $?$ & & 374.40 & \\
\hline & $\mathbf{R}$ & & 374.50 & \\
\hline & $\mathrm{N}$ & & 376.00 & \\
\hline & ? & & 376.25 & \\
\hline & $\mathrm{N}$ & & 376.50 & \\
\hline \multirow[t]{9}{*}{$41 X$} & $\mathrm{~N}$ & 380.20 & 382.70 & \\
\hline & $\mathrm{R}$ & & 382.90 & \\
\hline & $\mathrm{N}$ & & 384.90 & \\
\hline & $\mathrm{R}$ & & 385.10 & \\
\hline & $\mathrm{N}$ & & 387.20 & $\mathrm{R}$ at $386.20 \mathrm{mbsf}$ \\
\hline & $\mathrm{R}$ & & 387.75 & \\
\hline & $\mathrm{N}$ & & 388.35 & \\
\hline & $\mathrm{R}$ & & 388.55 & \\
\hline & $\mathrm{N}$ & & 390.00 & $\mathrm{R}$ at $389.45 \mathrm{mbsf}$ \\
\hline \multirow[t]{5}{*}{$42 \mathrm{X}$} & $\mathrm{N}$ & 390.00 & 392.10 & $\mathrm{R}$ at $390.10 \mathrm{mbsf}$ \\
\hline & $\mathrm{R}$ & & 392.40 & \\
\hline & $\mathrm{N}$ & & 392.80 & \\
\hline & $\mathrm{R}$ & & 393.10 & \\
\hline & $\mathrm{N}$ & & 393.25 & \\
\hline
\end{tabular}

125-783A-

$\begin{array}{lllll}\text { IR } & \text { N } & 0.00 & 1.50 & \\ & \mathrm{R} & & 1.60 & \\ & \mathrm{~N} & & 2.50 & \\ & ? & & 3.70 & \\ & \mathrm{~N} & & 5.20 & \\ & ? & & 6.20 & \\ & \mathrm{~N} & & 7.20 & \\ & ? & & 7.50 & \\ & \mathrm{~N} & & 9.00 & \mathrm{R} \text { trend at } 8.6 \text { mbsf } \\ & \mathrm{R} & 9.70 & 12.00 & \text { Good trends toward } \mathrm{R} \\ \mathrm{R} & \mathrm{R} & & & \text { No core } \\ \text { 3R } & \mathrm{R} & 26.00 & 32.50 & \text { Good trends toward R } \\ 5 \mathrm{R} & \mathrm{R} & 35.70 & 40.70 & \\ 6 \mathrm{R} & & & & \text { No core } \\ 7 \mathrm{R} & \mathrm{R} & 52.40 & 56.80 & \\ 8 \mathrm{R} & \mathrm{N} & 62.10 & 66.30 & \text { R at } 65.1 \text { mbsf }\end{array}$

125-784A-

\begin{tabular}{|c|c|c|c|c|}
\hline IR & & & & No core \\
\hline $2 \mathrm{R}$ & & & & No core \\
\hline \multirow[t]{7}{*}{$3 R$} & $\mathrm{~N}$ & 10.90 & 11.05 & \\
\hline & $\mathrm{R}$ & & 11.35 & \\
\hline & $\mathrm{N}$ & & 12.10 & \\
\hline & $?$ & & 12.40 & \\
\hline & $\mathrm{N}$ & & 14.70 & \\
\hline & $\mathbf{R}$ & & 15.30 & \\
\hline & $\mathrm{N}$ & & 15.90 & \\
\hline \multirow[t]{6}{*}{$4 R$} & $\mathrm{~N}$ & 20.40 & 20.80 & \\
\hline & $\mathrm{R}$ & & 21.10 & \\
\hline & $\mathrm{N}$ & & 21.30 & \\
\hline & $\mathrm{R}$ & & 21.80 & \\
\hline & $\mathrm{N}$ & & 22.40 & \\
\hline & $\mathrm{R}$ & & 25.60 & \\
\hline $5 \mathrm{R}$ & & & & No core \\
\hline \multirow[t]{9}{*}{$6 R$} & $\mathrm{R}$ & 39.60 & 42.75 & \\
\hline & $\mathrm{N}$ & & 43.70 & \\
\hline & & & 44.10 & No core \\
\hline & $\mathrm{N}$ & & 45.10 & \\
\hline & $\mathrm{R}$ & & 45.25 & \\
\hline & $\mathrm{N}$ & & 45.60 & \\
\hline & $\mathrm{R}$ & & 45.75 & \\
\hline & $\mathrm{N}$ & & 47.00 & \\
\hline & $\mathrm{R}$ & & 47.60 & \\
\hline $7 \mathrm{R}$ & $\mathrm{R}$ & 51.00 & 53.65 & \\
\hline \multirow[t]{2}{*}{$8 \mathrm{R}$} & $\mathrm{R}$ & 58.80 & 59.50 & \\
\hline & $\mathrm{N}$ & & 61.60 & \\
\hline
\end{tabular}

APPENDIX A (continued).

\begin{tabular}{|c|c|c|c|c|}
\hline \multirow[b]{2}{*}{ Core } & \multirow[b]{2}{*}{ Polarity } & \multicolumn{2}{|c|}{ Depth (mbsf) } & \multirow[b]{2}{*}{ Comments } \\
\hline & & Upper & Lower & \\
\hline & $\mathrm{R}$ & & 66.50 & \\
\hline 9R & $\mathrm{R}$ & 68.50 & 75.20 & \\
\hline IOR & $\mathrm{N}$ & 78.10 & 83.90 & \\
\hline $11 \mathrm{R}$ & & & & No core \\
\hline $12 \mathrm{R}$ & $\mathbf{R}$ & 97.40 & 100.20 & \\
\hline $13 \mathrm{R}$ & $\mathrm{R}$ & 106.50 & 107.50 & $\begin{array}{l}\text { Very short core: only } 5 \\
\text { measurements } \mathrm{N} \text { at } 119.70 \mathrm{mbsf}\end{array}$ \\
\hline $14 \mathrm{R}$ & $\mathrm{R}$ & 116.70 & 123.70 & \\
\hline $15 \mathrm{R}$ & $\mathrm{R}$ & 126.40 & 136.00 & \\
\hline $16 \mathrm{R}$ & $\mathrm{N}$ & 136.00 & 145.40 & ? at $142.3,143.6$, and $144.5 \mathrm{mbsf}$ \\
\hline \multirow[t]{3}{*}{$17 \mathrm{R}$} & $\mathrm{R}$ & 145.70 & 149.45 & \\
\hline & $\mathrm{N}$ & & 152.20 & \\
\hline & $\mathrm{R}$ & & 153.00 & \\
\hline \multirow[t]{3}{*}{$18 \mathrm{R}$} & $\mathrm{R}$ & 155.40 & 156.50 & \\
\hline & $\mathrm{N}$ & & 152.20 & \\
\hline & $\mathrm{R}$ & & 165.00 & $\begin{array}{l}\text { Subhorizontal bedding at } 163.93 \\
\text { mbsf }\end{array}$ \\
\hline \multirow[t]{2}{*}{$19 \mathrm{R}$} & $\mathrm{N}$ & 165.60 & 168.30 & \\
\hline & $?$ & & 168.50 & \\
\hline \multirow[t]{2}{*}{$20 \mathrm{R}$} & $\mathrm{R}$ & 174.40 & 175.75 & \\
\hline & $\mathrm{N}$ & & 182.10 & $\mathrm{R}$ at $176.75 \mathrm{mbsf}$ \\
\hline
\end{tabular}

125-786A-

\begin{tabular}{|c|c|c|c|c|}
\hline \multirow[t]{5}{*}{$1 \mathrm{H}$} & $\mathrm{N}$ & 0.0 & 0.35 & \\
\hline & $\mathbf{R}$ & & 2.20 & \\
\hline & $\mathrm{N}$ & & 2.50 & \\
\hline & $\mathbf{R}$ & & 4.65 & \\
\hline & $\mathrm{N}$ & & 9.00 & \\
\hline & $\mathbf{R}$ & & 9.30 & \\
\hline \multirow[t]{5}{*}{$2 \mathrm{H}$} & $\mathrm{N}$ & 9.7 & 11.35 & \\
\hline & $\mathrm{R}$ & & 13.15 & \\
\hline & $\mathrm{N}$ & & 15.40 & \\
\hline & $\mathrm{R}$ & & 17.60 & \\
\hline & $\mathrm{N}$ & & 19.15 & \\
\hline \multirow[t]{6}{*}{$3 \mathrm{H}$} & $\mathrm{R}$ & 19.20 & 20.75 & \\
\hline & $?$ & & 22.20 & No data \\
\hline & $\mathrm{N}$ & & 23.60 & \\
\hline & $\mathbf{R}$ & & 25.30 & \\
\hline & $\mathrm{N}$ & & 26.60 & \\
\hline & $\mathrm{R}$ & & 28.70 & \\
\hline \multirow[t]{2}{*}{$4 \mathrm{X}$} & $\mathrm{N}$ & 28.70 & 30.40 & \\
\hline & $\mathrm{R}$ & & 30.85 & \\
\hline $5 X$ & $\mathrm{~N}$ & 38.20 & 42.65 & $\mathrm{R}$ at 41.30 and $42.00 \mathrm{mbsf}$ \\
\hline \multirow[t]{3}{*}{$6 \mathrm{X}$} & $\mathrm{N}$ & 47.60 & 53.20 & \\
\hline & $\mathbf{R}$ & & 53.50 & $\begin{array}{l}\text { Cores } 5 \mathrm{X}, 6 \mathrm{X} \text {, and } 7 \mathrm{X} \text { have good } \\
\text { trend behavior }\end{array}$ \\
\hline & $\mathrm{N}$ & & 53.95 & \\
\hline $7 X$ & $\mathrm{~N}$ & 57.10 & 64.20 & \\
\hline $8 \mathrm{X}$ & $\mathrm{N}$ & 67.10 & 68.20 & \\
\hline \multirow[t]{15}{*}{$9 \mathrm{X}$} & $\mathrm{N}$ & 76.80 & 77.10 & \\
\hline & $\mathrm{R}$ & & 78.60 & \\
\hline & $\mathrm{N}$ & & 79.00 & \\
\hline & $\mathrm{R}$ & & 79.20 & \\
\hline & $?$ & & 79.75 & \\
\hline & $\mathrm{N}$ & & 80.00 & \\
\hline & $\mathrm{R}$ & & 81.00 & \\
\hline & $\mathrm{N}$ & & 81.30 & \\
\hline & $\mathrm{R}$ & & 81.65 & \\
\hline & $\mathrm{N}$ & & 82.40 & \\
\hline & $\mathrm{R}$ & & 83.20 & \\
\hline & $?$ & & 83.50 & \\
\hline & $\mathrm{N}$ & & 84.00 & \\
\hline & $\mathrm{R}$ & & 84.70 & \\
\hline & $\mathrm{N}$ & & 85.00 & \\
\hline \multirow[t]{2}{*}{$10 X$} & $\mathrm{~N}$ & 86.40 & 92.30 & \\
\hline & $?$ & & 93.80 & $\begin{array}{l}\text { No } 10-\mathrm{mT} \text { WCC data from this } \\
\text { section }\end{array}$ \\
\hline \multirow[t]{8}{*}{$11 x$} & $\mathrm{R}$ & 96.00 & 96.40 & \\
\hline & ? & & 96.60 & \\
\hline & $\mathrm{R}$ & & 96.90 & \\
\hline & ? & & 97.10 & \\
\hline & $\mathrm{R}$ & & 98.00 & \\
\hline & $\mathrm{N}$ & & 100.00 & \\
\hline & $?$ & & 100.15 & \\
\hline & $\mathrm{R}$ & & 100.60 & \\
\hline
\end{tabular}


APPENDIX A (continued).

\begin{tabular}{|c|c|c|c|c|}
\hline \multirow[b]{2}{*}{ Core } & \multirow[b]{2}{*}{ Polarity } & \multicolumn{2}{|c|}{ Depth (mbsf) } & \multirow[b]{2}{*}{ Comments } \\
\hline & & Upper & Lower & \\
\hline & $\mathrm{N}$ & & 101.80 & \\
\hline & $?$ & & 102.70 & \\
\hline & $\mathrm{N}$ & & 103.80 & \\
\hline & $?$ & & 104.00 & \\
\hline & $\mathrm{N}$ & & 104.20 & \\
\hline & $?$ & & 104.40 & \\
\hline
\end{tabular}


APPENDIX B

DSF Magnetometer Remanence Data for Leg 125 Samples

\begin{tabular}{|c|c|c|c|c|c|c|c|c|}
\hline \multirow[b]{2}{*}{$\begin{array}{l}\text { Core, section, } \\
\text { interval }(\mathrm{cm})\end{array}$} & \multirow[b]{2}{*}{$\begin{array}{l}\text { Depth } \\
\text { (mbsf) }\end{array}$} & \multicolumn{3}{|c|}{ Natural remanent magnetization } & \multicolumn{3}{|c|}{ Remagnetization at $40 \mathrm{mT}$} & \multirow[b]{2}{*}{ Polarity $^{\mathrm{a}}$} \\
\hline & & $\begin{array}{l}\text { Declination } \\
\text { (degrees) }\end{array}$ & $\begin{array}{c}\text { Inclination } \\
\text { (degrees) }\end{array}$ & $\begin{array}{r}\text { Intensity } \\
(\mathrm{mA} / \mathrm{m})\end{array}$ & $\begin{array}{c}\text { Declination } \\
\text { (degrees) }\end{array}$ & $\begin{array}{l}\text { Inclination } \\
\text { (degrees) }\end{array}$ & $\begin{array}{l}\text { Intensity } \\
(\mathrm{mA} / \mathrm{m})\end{array}$ & \\
\hline \multicolumn{9}{|l|}{$125-782 \mathrm{~A}-$} \\
\hline $1 \mathrm{H}-1,52$ & 0.52 & 332,4 & 55.1 & 57.1 & 325.3 & 70.7 & 19.1 & $\mathrm{~N}$ \\
\hline $1 \mathrm{H}-2,52$ & 2.02 & 352.2 & 57.0 & 84.2 & 345.7 & 47.9 & 21.1 & $\mathrm{~N}$ \\
\hline $1 \mathrm{H}-3,52$ & 3.52 & 349.8 & 61.2 & 109.3 & 340.7 & 65.5 & 28.8 & $\mathrm{~N}$ \\
\hline $1 \mathrm{H}-4,52$ & 5.02 & 0.4 & 55.1 & 28.9 & 339.8 & 46.0 & 2.7 & $\mathrm{~N}$ \\
\hline $1 \mathrm{H}-5,52$ & 6.52 & 56.7 & 76.1 & 54.4 & 250.0 & 77.3 & 5.0 & $\mathrm{~N}$ \\
\hline $1 \mathrm{H}-6,52$ & 8.02 & 107.5 & 13.8 & 33.3 & 137.2 & 13.7 & 9.1 & $?$ \\
\hline $1 \mathrm{H}-7,52$ & 9.52 & 103.1 & 37.3 & 57.6 & 121.7 & 40.1 & 14.7 & $\mathrm{~N}$ \\
\hline $2 \mathrm{H}-1,16$ & 9.96 & 231.1 & 36.1 & 30.8 & 259.4 & 28.1 & 20.0 & $\mathrm{~N}$ \\
\hline $2 \mathrm{H}-2,16$ & 11.46 & 296.6 & -46.2 & 2.6 & 276.1 & -26.7 & 1.1 & $\mathrm{R}$ \\
\hline $2 \mathrm{H}-3,16$ & 12.96 & 206.5 & 65.7 & 14.3 & 258.5 & 72.2 & 4.1 & $\mathrm{~N}$ \\
\hline $2 \mathrm{H}-4,16$ & 14.46 & 253.1 & 58.7 & 28.5 & 243.4 & 53.8 & 6.5 & $\mathrm{~N}$ \\
\hline $2 \mathrm{H}-5,16$ & 15.96 & 261.0 & 62.4 & 36.4 & 264.8 & 48.4 & 7.6 & $\mathrm{~N}$ \\
\hline $2 \mathrm{H}-6,16$ & 17.46 & 233.6 & 58.7 & 80.7 & 228.3 & 58.3 & 19.3 & $\mathrm{~N}$ \\
\hline $2 \mathrm{H}-7.16$ & 18.96 & 251.5 & 46.8 & 104.7 & 255.6 & 45.7 & 18.7 & $\mathrm{~N}$ \\
\hline $3 \mathrm{H}-1,16$ & 19.46 & 17.5 & 70.0 & 8.0 & 251.5 & -22.8 & 2.3 & $\mathrm{R}$ \\
\hline $3 \mathrm{H}-2,16$ & 20.96 & 160.2 & 53.2 & 10.2 & 186.6 & 57.9 & 0.7 & $\mathrm{~N}$ \\
\hline $3 \mathrm{H}-3,16$ & 22.46 & 208.4 & 41.8 & 18.8 & 174.6 & 10.5 & 3.8 & $?$ \\
\hline $3 \mathrm{H}-4,16$ & 23.96 & 208.7 & 52.1 & 99.5 & 215.2 & 52.2 & 26.9 & $\mathrm{~N}$ \\
\hline $3 \mathrm{H}-5,16$ & 25.46 & 252.4 & -4.8 & 7.2 & 42.0 & -49.7 & 4.0 & $\mathrm{R}$ \\
\hline $3 \mathrm{H}-6,16$ & 26.96 & 39.4 & -35.1 & 44.0 & 51.4 & -45.0 & 13.8 & $\mathrm{R}$ \\
\hline $3 \mathrm{H}-7,16$ & 28.46 & 31.4 & -44.6 & 31.3 & 57.0 & -61.5 & 9.6 & $\mathrm{R}$ \\
\hline $4 \mathrm{H}-1.19$ & 28.99 & 240.7 & 33.9 & 7.6 & 240.1 & 23.0 & 1.0 & $\mathrm{~N}$ \\
\hline $4 \mathrm{H}-2,19$ & 30.49 & 215.2 & -1.2 & 13.2 & 240.6 & 20.5 & 0.8 & $\mathrm{~N}$ \\
\hline $4 \mathrm{H}-3,115$ & 32.95 & 38.2 & 45.2 & 13.9 & 37.2 & 33.4 & 2.5 & $\mathrm{~N}$ \\
\hline $4 \mathrm{H}-4,19$ & 33.49 & 209.0 & 16.1 & 17.4 & 205.9 & 14.2 & 1.8 & ? \\
\hline $4 \mathrm{H}-5,19$ & 34.99 & 248.2 & -10.0 & 13.5 & 239.5 & 10.7 & 1.4 & ? \\
\hline $4 \mathrm{H}-6,19$ & 36.49 & 206.3 & -36.6 & 26.5 & 234.7 & -41.0 & 9.3 & $\mathrm{R}$ \\
\hline $4 \mathrm{H}-7,19$ & 37.99 & 227.7 & -52.1 & 26.4 & 235.9 & -59.4 & 7.1 & R \\
\hline $5 \mathrm{H}-1,37$ & 38.68 & 264.6 & 20.0 & 5.6 & 220.0 & -37.7 & 2.2 & $\mathrm{R}$ \\
\hline $5 \mathrm{H}-2,37$ & 40.18 & 153.8 & -9.5 & 14.6 & 150.0 & -40.0 & 2.4 & $\mathrm{R}$ \\
\hline $5 \mathrm{H}-3,37$ & 41.68 & 193.0 & -36.8 & 23.3 & 188.1 & -37.5 & 7.2 & $\mathrm{R}$ \\
\hline $5 \mathrm{H}-4,37$ & 43.18 & 219.2 & -8.8 & 5.9 & 172.1 & -15.4 & 3.8 & R \\
\hline $5 \mathrm{H}-5,37$ & 44.68 & 276.7 & -26.9 & 11.0 & 234.8 & -47.7 & 3.3 & $\mathrm{R}$ \\
\hline $5 \mathrm{H}-6,37$ & 46.18 & 219.1 & -37.4 & 54.6 & 209.6 & -37.8 & 12.9 & $\mathrm{R}$ \\
\hline $5 \mathrm{H}-7.16$ & 47.46 & 219.1 & -39.8 & 35.6 & 194.9 & -44.0 & 10.5 & $\mathrm{R}$ \\
\hline $6 \mathrm{H}-1.106$ & 48.86 & 161.5 & 61.3 & 10.6 & 165.0 & 58.6 & 2.8 & $\mathrm{~N}$ \\
\hline $6 \mathrm{H}-2,23$ & 49.53 & 193.1 & 80.4 & 9.4 & 169.0 & 61.0 & 3.6 & $\mathrm{~N}$ \\
\hline $6 \mathrm{H}-3,102$ & 51.82 & 194.0 & 42.6 & 75.0 & 194.4 & 43.6 & 16.3 & $\mathrm{~N}$ \\
\hline $6 \mathrm{H}-4,60$ & 52.90 & 212.5 & 56.3 & 99.0 & 203.0 & 60.8 & 25.3 & $\mathrm{~N}$ \\
\hline $6 \mathrm{H}-5,64$ & 54.44 & 357.3 & -36.7 & 32.1 & 29.0 & -54.0 & 23.9 & $\mathrm{R}$ \\
\hline $6 \mathrm{H}-6,69$ & 55.99 & 321.9 & -73.3 & 37.3 & 12.5 & -47.0 & 15.7 & $\mathrm{R}$ \\
\hline $6 \mathrm{H}-7,45$ & 57.25 & 6.8 & -49.1 & 8.2 & 11.9 & -78.4 & 3.0 & $\mathrm{R}$ \\
\hline $7 \mathrm{H}-1,44$ & 57.74 & 319.0 & -38.9 & 19.1 & 337.0 & -27.7 & 4.0 & $\mathrm{R}$ \\
\hline $7 \mathrm{H}-2,44$ & 59.24 & 284.5 & -65.6 & 7.8 & 350.0 & -64.5 & 1.3 & R \\
\hline $7 \mathrm{H}-3,44$ & 60.74 & 267.0 & -58.9 & 6.9 & 44.4 & -68.8 & 2.3 & $\mathrm{R}$ \\
\hline $7 \mathrm{H}-4,44$ & 62.24 & 185.8 & -76.6 & 7.8 & 97.6 & -45.0 & 3.4 & $\mathrm{R}$ \\
\hline $7 \mathrm{H}-5,44$ & 63.74 & 49.4 & -31.6 & 43.1 & 59.9 & -30.8 & 16.1 & $\mathrm{R}$ \\
\hline $7 \mathrm{H}-6,44$ & 65.24 & 253.5 & 16.7 & 50.9 & 209.6 & -50.8 & 6.4 & $\mathrm{R}$ \\
\hline $7 \mathrm{H}-7,44$ & 66.74 & 215.1 & -74.5 & 19.0 & 130.2 & -66.6 & 6.2 & R \\
\hline $8 \mathrm{H}-1,27$ & 67.07 & 247.5 & 38.5 & 44.9 & 347.4 & 50.3 & 3.0 & $\mathrm{~N}$ \\
\hline $8 \mathrm{H}-2,27$ & 68.57 & 126.5 & -4.3 & 17.4 & 206.4 & 17.0 & 1.9 & ? \\
\hline $8 \mathrm{H}-3,27$ & 70.07 & 353.6 & 23.0 & 36.9 & 38.9 & 15.5 & 4.3 & ? \\
\hline $8 \mathrm{H}-4,27$ & 71.57 & 250.8 & 18.8 & 20.4 & 137.6 & -55.6 & 3.8 & $\mathrm{R}$ \\
\hline $8 \mathrm{H}-5,27$ & 73.07 & 70.0 & -46.8 & 29.9 & 112.4 & -56.3 & 10.4 & $\mathrm{R}$ \\
\hline $8 \mathrm{H}-6,27$ & 74.57 & 233.8 & 74.0 & 36.7 & 224.0 & 61.4 & 10.1 & $\mathrm{~N}$ \\
\hline $8 \mathrm{H}-7,27$ & 76.07 & 42.6 & -28.5 & 29.3 & 186.0 & 44.8 & 4.0 & $\mathrm{~N}$ \\
\hline $9 \mathrm{H}-1,20$ & 76.50 & 336.2 & 37.3 & 30.5 & 341.0 & 34.7 & 8.6 & $\mathrm{~N}$ \\
\hline $9 \mathrm{H}-2,20$ & 78.00 & 328.8 & 65.8 & 39.0 & 353.8 & 48.6 & 8.5 & $\mathrm{~N}$ \\
\hline $9 \mathrm{H}-3,8$ & 79.38 & 303.4 & 39.7 & 25.4 & 319.0 & 32.0 & 6.3 & $\mathrm{~N}$ \\
\hline $9 \mathrm{H}-4,8$ & 80.88 & 297.9 & 61.1 & 35.8 & 303.8 & 63.3 & 7.7 & $\mathrm{~N}$ \\
\hline $9 \mathrm{H}-5,8$ & 82.38 & 144.3 & 73.3 & 21.0 & 31.1 & 45.1 & 9.2 & $\mathrm{~N}$ \\
\hline $9 \mathrm{H}-6,8$ & 83.88 & 46.7 & 60.8 & 46.7 & 214.1 & -16.9 & 2.7 & ? \\
\hline $11 X-1,24$ & 95.94 & 236.1 & -55.6 & 96.0 & 274.9 & -26.4 & 13.3 & $\mathrm{R}$ \\
\hline $11 X-2,24$ & 97.44 & 111.2 & -66.1 & 96.2 & 111.5 & -58.4 & 18.1 & $\mathrm{R}$ \\
\hline $11 X-3,12$ & 98.82 & 104.8 & 11.8 & 68.8 & 106.3 & 31.5 & 18.0 & $\mathrm{R}$ \\
\hline $12 X-1,60$ & 105.90 & 13.3 & -70.7 & 36.0 & 342.6 & -72.7 & 9.9 & $\mathrm{R}$ \\
\hline $12 X-2,53$ & 107.33 & 90.3 & -47.0 & 50.1 & 96.2 & -47.2 & 16.5 & $\mathrm{R}$ \\
\hline $12 X-3,52$ & 108.83 & 140.0 & -68.8 & 68.9 & 154.1 & -71.9 & 22.7 & $\mathrm{R}$ \\
\hline $13 \mathrm{X}-1,109$ & 116.09 & 73.6 & -30.3 & 8.3 & 61.4 & -3.4 & 1.2 & ? \\
\hline $13 \mathrm{X}-2,19$ & 116.69 & 330.1 & -77.9 & 17.9 & 209.2 & -28.7 & 1.5 & R \\
\hline $13 \mathrm{X}-2,65$ & 117.05 & 307.1 & 38.7 & 62.2 & 313.7 & 46.6 & 19.9 & $\mathrm{~N}$ \\
\hline
\end{tabular}


APPENDIX B (continued).

\begin{tabular}{|c|c|c|c|c|c|c|c|c|}
\hline \multirow[b]{2}{*}{$\begin{array}{l}\text { Core, section, } \\
\text { interval }(\mathrm{cm})\end{array}$} & \multirow[b]{2}{*}{$\begin{array}{l}\text { Depth } \\
\text { (mbsf) }\end{array}$} & \multicolumn{3}{|c|}{ Natural remanent magnetization } & \multicolumn{3}{|c|}{ Remagnetization at $40 \mathrm{~m} \mathrm{~T}$} & \multirow[b]{2}{*}{ Polarity } \\
\hline & & $\begin{array}{c}\text { Declination } \\
\text { (degrees) }\end{array}$ & $\begin{array}{c}\text { Inclination } \\
\text { (degrees) }\end{array}$ & $\begin{array}{r}\text { Intensity } \\
(\mathrm{mA} / \mathrm{m})\end{array}$ & $\begin{array}{c}\text { Declination } \\
\text { (degrees) }\end{array}$ & $\begin{array}{l}\text { Inclination } \\
\text { (degrees) }\end{array}$ & $\begin{array}{l}\text { Intensity } \\
(\mathrm{mA} / \mathrm{m})\end{array}$ & \\
\hline $13 \mathrm{X}-3,81$ & 118.81 & 14.1 & -34.0 & 39.5 & 6.1 & -31.2 & 7.5 & $\mathrm{R}$ \\
\hline $13 X-4,9$ & 119.59 & 41.5 & -46.4 & 22.6 & 49.7 & -43.4 & 3.8 & $\mathbf{R}$ \\
\hline $14 \mathrm{X}-1,28$ & 124.88 & 216.3 & -61.8 & 39.8 & 215.3 & -62.7 & 11.3 & $\mathbf{R}$ \\
\hline $14 X-2,28$ & 126.38 & 207.0 & 51.6 & 28.4 & 214.8 & 47.2 & 10.3 & $\mathrm{~N}$ \\
\hline $14 X-3,28$ & 127.88 & 207.5 & 44.2 & 40.8 & 187.4 & 43.2 & 17.6 & $\mathrm{~N}$ \\
\hline $14 X-4,28$ & 129.38 & 135.5 & -43.8 & 30.6 & 138.3 & -45.8 & 7.0 & $\mathrm{R}$ \\
\hline $15 X-1,21$ & 134.51 & 332.5 & -29.5 & 24.7 & 287.2 & 41.7 & 10.2 & $\mathrm{~N}$ \\
\hline $15 X-2,21$ & 136.01 & 320.6 & 24.1 & 53.9 & 306.3 & 54.0 & 22.0 & $\mathrm{~N}$ \\
\hline $15 X-3,21$ & 137.51 & 45.7 & -69.3 & 60.2 & 144.0 & -52.7 & 16.1 & $\mathrm{R}$ \\
\hline $16 \mathrm{X}-1,22$ & 144.12 & 264.5 & 27.3 & 37.0 & 268.1 & 32.6 & 12.0 & $\mathrm{~N}$ \\
\hline $16 \mathrm{X}-2,22$ & 145.62 & 143.1 & 33.6 & 80.0 & 125.3 & 49.7 & 27.2 & $\mathrm{~N}$ \\
\hline $16 \mathrm{X}-3,22$ & 147.12 & 273.9 & 39.5 & 43.3 & 288.2 & 49.4 & 12.9 & $\mathrm{~N}$ \\
\hline $17 X-1,46$ & 154.06 & 134.1 & -28.1 & 23.1 & 262.3 & -35.5 & 2.4 & $\mathrm{R}$ \\
\hline $17 X-2,46$ & 155.56 & 136.5 & -42.4 & 39.3 & 161.5 & -41.0 & 9.0 & $\mathrm{R}$ \\
\hline $17 X-3,46$ & 157.06 & 348.0 & -46.0 & 26.3 & 13.3 & -53.0 & 4.9 & $\mathrm{R}$ \\
\hline $17 X-4,46$ & 158.56 & 12.8 & -62.7 & 43.7 & 341.4 & -62.6 & 11.3 & $\mathrm{R}$ \\
\hline $17 X-5,46$ & 160.06 & 83.7 & -69.7 & 24.6 & 62.0 & -65.0 & 4.8 & $\mathrm{R}$ \\
\hline $18 X-1,60$ & 163.80 & 339.9 & 8.7 & 18.9 & 335.8 & 46.7 & 8.6 & $\mathrm{~N}$ \\
\hline $18 X-2,60$ & 165.30 & 44.5 & -55.3 & 71.5 & 150.9 & 58.4 & 15.4 & $\mathrm{~N}$ \\
\hline $19 X-1,80$ & 173.70 & 219.7 & 12.3 & 29.3 & 225.6 & 32.3 & 11.4 & $\mathrm{~N}$ \\
\hline $19 X-2,80$ & 175.20 & 276.2 & 37.6 & 39.4 & 266.4 & 44.0 & 13.7 & $\mathrm{~N}$ \\
\hline $19 X-3,80$ & 176.70 & 132.0 & 45.3 & 34.8 & 137.8 & 55.4 & 11.0 & $\mathrm{~N}$ \\
\hline $19 X-4,22$ & 177.62 & 19.1 & 50.3 & 8.8 & 320.6 & 65.3 & 5.4 & $\mathrm{~N}$ \\
\hline $20 \mathrm{X}-1,55$ & 183.05 & 78.8 & -20.1 & 45.5 & 145.2 & -45.2 & 22.6 & $\mathbf{R}$ \\
\hline $21 X-1,103$ & 193.13 & 359.2 & -55.2 & 102.2 & 340.9 & -43.2 & 22.5 & $\mathrm{R}$ \\
\hline $21 X-2,103$ & 194.63 & 121.6 & -73.5 & 61.4 & 191.6 & -55.1 & 15.3 & $\mathrm{R}$ \\
\hline $21 X-3,103$ & 196.13 & 186.7 & 41.7 & 71.4 & 184.1 & 49.0 & 25.5 & $\mathrm{~N}$ \\
\hline $21 X-4,103$ & 197.63 & 68.8 & 3.0 & 34.3 & 74.7 & 49.7 & 12.6 & $\mathrm{~N}$ \\
\hline $21 X-5,83$ & 198.93 & 347.2 & -9.6 & 70.4 & 331.6 & 41.7 & 19.3 & $\mathrm{~N}$ \\
\hline $22 X-1,55$ & 202.60 & 248.6 & -74.2 & 33.1 & 209.4 & -63.4 & 11.0 & $\mathrm{R}$ \\
\hline $22 X-2,33$ & 203.98 & 38.7 & -30.1 & 29.5 & 35.4 & -36.2 & 9.7 & $\mathrm{R}$ \\
\hline $23 \mathrm{X}-1,91$ & 212.41 & 112.2 & 42.4 & 28.9 & 104.1 & 47.6 & 13.4 & $\mathrm{~N}$ \\
\hline $23 X-2,61$ & 213.61 & 11.2 & 36.6 & 143.0 & 1.0 & 47.4 & 44.1 & $\mathrm{~N}$ \\
\hline $23 \mathrm{X}-3,82$ & 215.32 & 47.3 & -51.3 & 17.7 & 38.5 & -38.0 & 4.5 & $\mathrm{R}$ \\
\hline $23 X-4,68$ & 216.68 & 216.6 & 41.3 & 33.3 & 226.4 & 50.1 & 8.5 & $\mathrm{~N}$ \\
\hline $23 X-5,58$ & 218.08 & 64.3 & 14.0 & 72.0 & 84.0 & 32.5 & 13.5 & $\mathrm{~N}$ \\
\hline $23 X-6,72$ & 219.72 & 11.1 & -60.2 & 33.3 & 234.6 & 32.0 & 10.5 & $\mathrm{~N}$ \\
\hline $23 X-7,7$ & 220.57 & 69.7 & 82.0 & 110.5 & 253.0 & 54.7 & 16.9 & $\mathrm{~N}$ \\
\hline $24 X-1,96$ & 221.96 & 277.8 & 61.5 & 76.5 & 267.8 & 58.0 & 22.4 & $\mathrm{~N}$ \\
\hline $24 X-2,106$ & 223.56 & 159.9 & 48.2 & 51.9 & 182.0 & 49.4 & 19.5 & $\mathrm{~N}$ \\
\hline $25 \mathrm{X}-1,96$ & 231.66 & 319.9 & -58.0 & 45.8 & 326.5 & -51.7 & 15.0 & $\mathrm{R}$ \\
\hline $25 X-2,60$ & 232.80 & 129.9 & -76.6 & 27.9 & 121.3 & -75.1 & 10.1 & $\mathrm{R}$ \\
\hline $25 X-3,80$ & 234.50 & 73.1 & -55.0 & 34.0 & 79.9 & -47.5 & 8.6 & $\mathrm{R}$ \\
\hline $25 X-4,95$ & 236.15 & 320.0 & -24.6 & 31.1 & 151.4 & 54.0 & 1.6 & $?$ \\
\hline $25 X-5,56$ & 237.26 & 149.7 & 44.5 & 38.3 & 156.6 & 68.3 & 9.2 & $\mathrm{~N}$ \\
\hline $25 \mathrm{X}-6,59$ & 238.79 & 333.9 & 37.1 & 45.1 & 325.8 & 44.8 & 14.4 & $\mathrm{~N}$ \\
\hline $26 \mathrm{X}-1,9$ & 240.39 & 127.5 & -65.7 & 40.5 & 127.2 & -47.2 & 7.7 & $\mathbf{R}$ \\
\hline $26 X-2,73$ & 242.53 & 319.3 & 31.3 & 27.0 & 295.7 & 27.8 & 8.8 & $\mathrm{~N}$ \\
\hline $26 \mathrm{X}-3,126$ & 244.56 & 35.2 & -5.8 & 7.0 & 189.4 & 64.3 & 0.4 & $?$ \\
\hline $26 X-4,107$ & 245.87 & 140.3 & 57.5 & 30.9 & 303.4 & 71.0 & 6.8 & $\mathrm{~N}$ \\
\hline $26 X-5,118$ & 247.48 & 121.1 & 37.0 & 59.4 & 115.4 & 43.0 & 12.7 & $\mathrm{~N}$ \\
\hline $26 \mathrm{X}-6,127$ & 249.07 & 279.6 & 31.8 & 12.9 & 263.5 & 60.2 & 5.8 & $\mathrm{~N}$ \\
\hline $27 X-1,117$ & 251.17 & 110.6 & -53.8 & 76.7 & 49.8 & 46.4 & 7.3 & $\mathrm{~N}$ \\
\hline $27 X-2,134$ & 252.84 & 272.4 & -3.9 & 45.5 & 260.4 & 13.3 & 11.6 & $\mathrm{~N}$ \\
\hline $27 X-3,56$ & 253.56 & 4.5 & -21.8 & 25.8 & 342.9 & -2.7 & 5.6 & $?$ \\
\hline $28 \mathrm{X}-1,59$ & 260.19 & 354.8 & -57.7 & 45.6 & 16.1 & -58.7 & 10.7 & $\mathrm{R}$ \\
\hline $28 X-2,24$ & 261.34 & 78.4 & -13.0 & 86.1 & 56.0 & -17.0 & 23.1 & $\mathrm{R}$ \\
\hline $28 X-3,49$ & 263.09 & 258.2 & 47.1 & 138.4 & 262.9 & 52.2 & 39.0 & $\mathrm{~N}$ \\
\hline $28 \times-4,37$ & 274.63 & 136.0 & -38.0 & 22.3 & 56.3 & -23.8 & 4.4 & $\mathbf{R}$ \\
\hline $28 X-6,105$ & 268.15 & 348.7 & -54.6 & 31.1 & 351.0 & -63.0 & 11.0 & $\mathbf{R}$ \\
\hline $29 X-1,16$ & 269.36 & 85.9 & -57.3 & 96.1 & 108.9 & -59.4 & 12.9 & $\mathrm{R}$ \\
\hline $29 \mathrm{X}-2,28$ & 270.98 & 106.7 & -68.8 & 46.6 & 220.7 & -56.2 & 11.0 & $\mathbf{R}$ \\
\hline $29 X-3,7$ & 272.27 & 104.2 & -78.3 & 24.9 & 140.6 & -63.2 & 7.2 & $\mathrm{R}$ \\
\hline $29 X-4,93$ & 274.63 & 270.0 & 25.9 & 39.0 & 286.4 & 56.3 & 16.9 & $\mathrm{~N}$ \\
\hline $29 X-5,9$ & 275.29 & 101.1 & 21.1 & 75.3 & 88.8 & 33.3 & 14.2 & $\mathrm{~N}$ \\
\hline $29 X-6,78$ & 277.48 & 302.6 & -76.7 & 60.4 & 259.3 & -45.7 & 27.1 & $\mathrm{R}$ \\
\hline $30 X-1,41$ & 279.21 & 188.7 & 23.1 & 159.5 & 180.3 & -47.5 & 60.6 & $\mathrm{R}$ \\
\hline $30 X-1,61$ & 279.41 & 159.4 & 18.3 & 163.1 & 180.5 & 43.8 & 52.5 & $\mathrm{~N}$ \\
\hline $30 X-2,61$ & 280.36 & 342.1 & -6.4 & 93.2 & 308.2 & 17.0 & 10.1 & $\mathrm{~N}$ \\
\hline $30 \mathrm{X}-\mathrm{CC}, 6$ & 282.06 & 15.7 & -23.7 & 77.1 & 193.2 & -77.5 & 8.1 & $\mathrm{R}$ \\
\hline $31 X-2,112$ & 290.10 & 87.4 & -34.1 & 19.3 & 98.0 & 18.4 & 4.2 & $\mathrm{~N}$ \\
\hline $31 X-3,46$ & 290.94 & 72.0 & -53.6 & 56.8 & 98.0 & -50.2 & 17.5 & $\mathrm{R}$ \\
\hline $32 X-1,132$ & 295.32 & 115.5 & -59.2 & 106.6 & 136.3 & -53.2 & 46.7 & $\mathrm{R}$ \\
\hline $32 X-2,90$ & 296.40 & 29.6 & -24.4 & 19.6 & 240.7 & -45.9 & 9.2 & $\mathrm{R}$ \\
\hline $32 X-4,37$ & 298.87 & 329.2 & 33.6 & 194.5 & 331.4 & 40.7 & 54.0 & $\mathrm{~N}$ \\
\hline
\end{tabular}


APPENDIX B (continued).

\begin{tabular}{|c|c|c|c|c|c|c|c|c|}
\hline \multirow[b]{2}{*}{$\begin{array}{l}\text { Core, section, } \\
\text { interval }(\mathrm{cm})\end{array}$} & \multirow[b]{2}{*}{$\begin{array}{l}\text { Depth } \\
\text { (mbsf) }\end{array}$} & \multicolumn{3}{|c|}{ Natural remanent magnetization } & \multicolumn{3}{|c|}{ Remagnetization at $40 \mathrm{mT}$} & \multirow[b]{2}{*}{ Polarity } \\
\hline & & $\begin{array}{c}\text { Declination } \\
\text { (degrees) }\end{array}$ & $\begin{array}{c}\text { Inclination } \\
\text { (degrees) }\end{array}$ & $\begin{array}{r}\text { Intensity } \\
(\mathrm{mA} / \mathrm{m})\end{array}$ & $\begin{array}{c}\text { Declination } \\
\text { (degrees) }\end{array}$ & $\begin{array}{l}\text { Inclination } \\
\text { (degrees) }\end{array}$ & $\begin{array}{l}\text { Intensity } \\
(\mathrm{mA} / \mathrm{m})\end{array}$ & \\
\hline $32 X-5,65$ & 300.65 & 3.6 & -55.6 & 36.2 & 254.8 & -40.7 & 14.0 & $\mathbf{R}$ \\
\hline $32 X-6,56$ & 302.06 & 119.1 & -58.9 & 90.0 & 79.8 & -64.2 & 34.3 & $\mathbf{R}$ \\
\hline $33 \mathrm{X}-1,57$ & 304.07 & 300.4 & 73.5 & 156.3 & 168.6 & 53.9 & 24.3 & $\mathrm{~N}$ \\
\hline $33 \mathrm{X}-2,13$ & 305.13 & 220.7 & 37.5 & 146.4 & 223.7 & 29.9 & 46.1 & $\mathrm{~N}$ \\
\hline $33 \mathrm{X}-3,53$ & 307.03 & 17.1 & 59.1 & 145.3 & 235.3 & 28.7 & 21.7 & $\mathrm{~N}$ \\
\hline $33 X-4,52$ & 308.52 & 315.7 & 16.9 & 83.8 & 315.3 & 9.0 & 16.8 & $?$ \\
\hline $33 X-5,53$ & 310.03 & 199.1 & -73.0 & 54.1 & 218.3 & -72.0 & 41.4 & R \\
\hline $33 X-6,37$ & 311.37 & 262.9 & 4.3 & 87.0 & 232.5 & 18.8 & 9.4 & $\mathrm{~N}$ \\
\hline $34 X-1,62$ & 313.72 & 12.0 & 19.5 & 164.4 & 351.0 & 6.6 & 9.6 & ? \\
\hline $35 X-1,92$ & 323.42 & 9.8 & -15.4 & 136.5 & 3.2 & -65.3 & 18.2 & $\mathrm{R}$ \\
\hline $35 X-2,42$ & 324.42 & 168.5 & -37.7 & 8.6 & 103.5 & -43.5 & 4.6 & $\mathrm{R}$ \\
\hline $35 X-3,38$ & 325.88 & 124.6 & 37.1 & 398.5 & 37.1 & 44.7 & 9.2 & $?$ \\
\hline $35 X-4,44$ & 327.44 & 81.5 & -30.2 & 30.8 & 49.1 & -51.2 & 16.7 & $\mathrm{R}$ \\
\hline $35 X-5,93$ & 329.43 & 193.9 & 57.9 & 79.9 & 278.2 & 33.5 & 4.4 & $\mathrm{~N}$ \\
\hline $35 X-6,75$ & 330.75 & 20.2 & 47.6 & 53.4 & 97.3 & 89.1 & 53.7 & ? \\
\hline $36 \mathrm{X}-1,111$ & 333.21 & 95.7 & 50.6 & 225.3 & 51.3 & 27.4 & 16.8 & $\mathrm{~N}$ \\
\hline $36 \mathrm{X}-2,111$ & 334.71 & 89.8 & 17.7 & 103.3 & 131.8 & 37.1 & 26.4 & $\mathrm{~N}$ \\
\hline $36 X-3,128$ & 336.38 & 358.6 & -73.9 & 46.2 & 195.0 & -53.3 & 12.8 & $\mathrm{R}$ \\
\hline $36 \mathrm{X}-4,113$ & 337.73 & 270.5 & -21.0 & 41.6 & 249.4 & -40.9 & 17.9 & $\mathrm{R}$ \\
\hline $36 \mathrm{X}-5,22$ & 338.32 & 45.6 & -52.3 & 57.9 & 53.6 & -43.1 & 15.4 & $\mathrm{R}$ \\
\hline $37 X-1,50$ & 342.10 & 214.7 & 53.9 & 90.6 & 232.3 & 48.3 & 24.5 & $\mathrm{~N}$ \\
\hline $37 X-2,93$ & 344.03 & 357.6 & 53.2 & 145.9 & 17.3 & 46.9 & 32.1 & $\mathrm{~N}$ \\
\hline $37 X-3,18$ & 344.78 & 353.9 & 0.1 & 190.2 & 179.5 & -52.8 & 11.9 & $\mathrm{R}$ \\
\hline $37 X-4,16$ & 346.26 & 1.5 & -35.8 & 66.8 & 303.2 & -57.0 & 34.1 & $\mathrm{R}$ \\
\hline $37 X-5,26$ & 347.86 & 64.8 & 30.4 & 93.8 & 89.2 & -15.0 & 7.8 & $\mathrm{R}$ \\
\hline $37 X-6,92$ & 350.02 & 290.1 & 3.3 & 26.5 & 158.5 & -36.5 & 13.9 & $\mathrm{R}$ \\
\hline $39 X-1,133$ & 363.01 & 334.4 & 6.5 & 59.6 & 258.6 & -36.5 & 13.9 & $\mathrm{R}$ \\
\hline $39 X-C C, 10$ & 363.28 & 316.0 & -53.7 & 42.6 & 359.4 & -4.8 & 6.8 & ? \\
\hline $40 X-3,52$ & 374.02 & 243.7 & -0.4 & 44.3 & 183.0 & -52.3 & 5.7 & $\mathrm{R}$ \\
\hline $40 X-4,59$ & 375.59 & 349.3 & 32.4 & 56.9 & 313.1 & 26.9 & 10.9 & $?$ \\
\hline $41 X-1,64$ & 380.84 & 92.7 & 18.1 & 133.1 & 115.3 & -0.3 & 0.9 & ? \\
\hline $41 X-2,24$ & 381.94 & 186.5 & -11.3 & 135.8 & 179.4 & 34.9 & 12.2 & $\mathrm{~N}$ \\
\hline $41 X-3,47$ & 383.67 & 98.2 & -32.2 & 55.8 & 17.4 & -76.9 & 5.8 & $\mathrm{R}$ \\
\hline $41 X-6,41$ & 388.11 & 84.0 & 21.6 & 83.3 & 40.0 & -54.5 & 6.0 & $\mathrm{R}$ \\
\hline $41 X-7,11$ & 389.31 & 234.1 & 36.1 & 9.8 & 254.9 & 19.9 & 2.6 & $\mathrm{~N}$ \\
\hline $41 X-C C, 5$ & 389.60 & 316.9 & -21.6 & 11.3 & 304.1 & -35.8 & 3.0 & $\mathrm{R}$ \\
\hline $42 X-1,58$ & 390.38 & 42.6 & 40.0 & 15.3 & 330.0 & -31.5 & 1.6 & ? \\
\hline $42 X-2,107$ & 392.37 & 53.7 & 45.8 & 19.9 & 335.2 & 31.1 & 2.0 & ? \\
\hline \multicolumn{9}{|l|}{$125-783 \mathrm{~A}-$} \\
\hline $1 \mathrm{R}-1,103$ & 1.03 & 325.4 & 30.0 & 77.3 & 347.6 & 53.7 & 17.6 & $\mathrm{~N}$ \\
\hline IR- 2,33 & 1.83 & 0.5 & 15.5 & 71.4 & 19.0 & 26.2 & 24.6 & $\mathrm{~N}$ \\
\hline IR-3, 6 & 3.06 & 343.0 & 60.2 & 63.6 & 330.2 & 64.0 & 10.5 & $\mathrm{~N}$ \\
\hline $1 \mathrm{R}-4,11$ & 4.61 & 63.0 & 74.7 & 101.0 & 82.5 & 62.9 & 16.2 & $\mathrm{~N}$ \\
\hline IR-5. 86 & 6.86 & 302.4 & 62.3 & 90.7 & 293.4 & 62.8 & 20.5 & $\mathrm{~N}$ \\
\hline 1R-6, 89 & 8.39 & 344.5 & 47.8 & 73.3 & 340.1 & 50.6 & 17.0 & $\mathrm{~N}$ \\
\hline $1 \mathrm{R}-7,45$ & 9.45 & 333.0 & 74.0 & 7.6 & 269.7 & 13.9 & 1.9 & ? \\
\hline $2 \mathrm{R}-\mathrm{I}, 52$ & 10.22 & 24.7 & -54.2 & 32.7 & 350.7 & -57.1 & 13.0 & $\mathrm{R}$ \\
\hline $2 \mathrm{R}-2,3$ & 11.23 & 252.1 & -55.0 & 44.2 & 253.4 & -54.4 & 12.2 & $\mathrm{R}$ \\
\hline $4 \mathrm{R}-1,114$ & 27.14 & 33.0 & -48.0 & 25.7 & 35.2 & -74.2 & 7.2 & $\mathrm{R}$ \\
\hline $4 R-2,113$ & 28.63 & 227.7 & 28.5 & 14.6 & 86.7 & -28.6 & 0.8 & $\mathrm{R}$ \\
\hline $4 \mathrm{R}-3,113$ & 30.13 & 220.2 & -32.5 & 23.1 & 210.6 & -32.7 & 6.3 & $\mathrm{R}$ \\
\hline $4 \mathrm{R}-4,113$ & 31.63 & 238.6 & -79.1 & 34.5 & 97.1 & -29.4 & 16.1 & $\mathrm{R}$ \\
\hline $4 R-5,40$ & 32.40 & 92.0 & -19.0 & 62.2 & 230.8 & -77.4 & 11.9 & R \\
\hline $5 \mathrm{R}-1,26$ & 35.96 & 171.4 & -82.9 & 47.3 & 87.0 & -61.6 & 10.0 & $\mathrm{R}$ \\
\hline $5 R-2,26$ & 37.46 & 190.9 & -75.8 & 60.5 & 188.9 & -67.2 & 23.4 & $\mathrm{R}$ \\
\hline SR-3. 26 & 38.96 & 87.1 & -51.7 & 99.2 & 106.4 & -52.5 & 28.4 & R \\
\hline $5 R-4,13$ & 40.33 & 262.2 & -50.7 & 67.1 & 283.2 & -51.9 & 11.6 & $\mathrm{R}$ \\
\hline $7 \mathrm{R}-1,90$ & 53.30 & 122.7 & -67.7 & 68.9 & 111.2 & -56.2 & 18.1 & $\mathrm{R}$ \\
\hline $7 R-2,90$ & 54.80 & 110.5 & -53.6 & 82.5 & 118.3 & -71.2 & 21.1 & $\mathrm{R}$ \\
\hline $7 \mathrm{R}-3,90$ & 56.30 & 182.1 & -64.3 & 84.6 & 193.3 & -65.2 & 21.8 & $\mathrm{R}$ \\
\hline $7 \mathrm{R}-4,3$ & 56.93 & 339.0 & -46.2 & 118.0 & 335.4 & -49.7 & 27.9 & $\mathrm{R}$ \\
\hline $8 \mathrm{R}-1,120$ & 63.30 & 262.8 & 53.7 & 123.0 & 268.6 & 53.4 & 35.4 & $\mathrm{~N}$ \\
\hline $8 \mathrm{R}-2,120$ & 64.80 & 86.5 & 55.3 & 48.7 & 99.3 & 56.1 & 13.6 & $\mathrm{~N}$ \\
\hline $8 \mathrm{R}-3,91$ & 66.01 & 278.8 & 44.5 & 41.6 & 303.3 & 27.0 & 13.8 & $\mathrm{~N}$ \\
\hline \multicolumn{9}{|l|}{$125-784 \mathrm{~A}-$} \\
\hline $1 R-1,68$ & 0.68 & 225.8 & -50.1 & 50.5 & 237.6 & -45.1 & 7.9 & $\mathbf{R}$ \\
\hline IR-CC, 17 & 1.11 & 279.3 & 42.2 & 63.3 & 274.3 & 48.2 & 12.4 & $\mathrm{~N}$ \\
\hline $3 \mathrm{R}-1,85$ & 11.75 & 150.5 & 40.1 & 37.9 & 126.6 & 42.8 & 9.4 & $\mathrm{~N}$ \\
\hline $3 \mathrm{R}-2,85$ & 13.25 & 79.2 & 45.3 & 86.0 & 65.4 & 35.7 & 17.3 & $\mathrm{~N}$ \\
\hline $3 \mathrm{R}-3,85$ & 14.75 & 67.7 & 42.6 & 124.2 & 41.9 & 61.7 & 25.4 & $\mathrm{~N}$ \\
\hline $3 R-4,85$ & 15.65 & 202.5 & 45.3 & 150.6 & 205.0 & 44.2 & 27.5 & $\mathrm{~N}$ \\
\hline
\end{tabular}


APPENDIX B (continued).

\begin{tabular}{|c|c|c|c|c|c|c|c|c|}
\hline \multirow[b]{2}{*}{$\begin{array}{l}\text { Core, section, } \\
\text { interval }(\mathrm{cm})\end{array}$} & \multirow[b]{2}{*}{$\begin{array}{l}\text { Depth } \\
\text { (mbsf) }\end{array}$} & \multicolumn{3}{|c|}{ Natural remanent magnetization } & \multicolumn{3}{|c|}{ Remagnetization at $40 \mathrm{mT}$} & \multirow[b]{2}{*}{ Polarity ${ }^{a}$} \\
\hline & & $\begin{array}{l}\text { Declination } \\
\text { (degrees) }\end{array}$ & $\begin{array}{l}\text { Inclination } \\
\text { (degrees) }\end{array}$ & $\begin{array}{l}\text { Intensity } \\
(\mathrm{mA} / \mathrm{m})\end{array}$ & $\begin{array}{l}\text { Declination } \\
\text { (degrees) }\end{array}$ & $\begin{array}{l}\text { Inclination } \\
\text { (degrees) }\end{array}$ & $\begin{array}{l}\text { Intensity } \\
(\mathrm{mA} / \mathrm{m})\end{array}$ & \\
\hline $4 \mathrm{R}-1,60$ & 21.00 & 309.6 & 13.0 & 38.2 & 15.4 & -23.8 & 25.6 & ${ }^{b} \mathrm{R}$ \\
\hline $4 \mathrm{R}-2,97$ & 22.87 & 312.6 & -37.4 & 57.2 & 314.1 & -45.7 & 21.1 & $\mathrm{R}$ \\
\hline $4 \mathrm{R}-3,97$ & 24.37 & 51.9 & -33.7 & 54.4 & 53.9 & -40.6 & 18.8 & $\mathrm{R}$ \\
\hline $4 R-4,59$ & 25.49 & 160.0 & 30.9 & 42.3 & 157.6 & 36.6 & 5.7 & $\mathrm{~N}$ \\
\hline $6 \mathrm{R}-1,60$ & 40.10 & 306.5 & -55.6 & 67.5 & 310.2 & -59.3 & 19.0 & $\mathrm{R}$ \\
\hline $6 \mathrm{R}-2,57$ & 41.57 & 162.4 & -48.1 & 119.1 & 155.2 & -34.7 & 21.7 & $\mathbf{R}$ \\
\hline $6 \mathrm{R}-3,52$ & 43.02 & 215.8 & 31.4 & 38.9 & 218.2 & 39.0 & 11.2 & $\mathrm{~N}$ \\
\hline $6 \mathrm{R}-4,69$ & 44.69 & 7.5 & 52.1 & 65.8 & 9.9 & 53.4 & 19.2 & $\mathrm{~N}$ \\
\hline $6 \mathrm{R}-5,51$ & 46.01 & 196.7 & -15.6 & 19.1 & 192.4 & -28.1 & 7.1 & $\mathrm{R}$ \\
\hline $6 \mathrm{R}-6,58$ & 47.58 & 344.3 & -23.2 & 25.0 & 331.8 & -19.8 & 9.8 & $\mathrm{R}$ \\
\hline $7 \mathrm{R}-1,39$ & 49.49 & 26.3 & -23.5 & 30.3 & 25.9 & -22.4 & 20.9 & $\mathrm{R}$ \\
\hline $7 \mathrm{R}-2,109$ & 51.69 & 350.0 & -12.3 & 51.2 & 342.4 & -23.9 & 15.7 & $\mathrm{R}$ \\
\hline $7 \mathrm{R}-3,112$ & 53.22 & 206.4 & -25.8 & 44.7 & 215.4 & -32.4 & 11.5 & $\mathrm{R}$ \\
\hline $8 \mathrm{R}-1,82$ & 59.62 & 278.7 & 27.8 & 18.8 & 294.5 & 29.8 & 5.1 & $\mathrm{~N}$ \\
\hline $8 \mathrm{R}-2,82$ & 61.12 & 185.4 & 6.6 & 33.1 & 180.4 & -3.4 & 8.3 & R \\
\hline $8 \mathrm{R}-3,82$ & 62.62 & 63.7 & -40.8 & 145.8 & 59.3 & -41.0 & 37.6 & $\mathrm{R}$ \\
\hline $8 R-4,82$ & 64.12 & 261.3 & -23.9 & 109.2 & 268.5 & -27.9 & 30.2 & $\mathbf{R}$ \\
\hline $8 \mathrm{R}-5,82$ & 65.62 & 34.6 & -13.2 & 61.3 & 26.8 & -26.8 & 15.2 & $\mathrm{R}$ \\
\hline $8 \mathrm{R}-6,8$ & 66.38 & 217.5 & -31.9 & 95.6 & 224.1 & -39.0 & 29.1 & $\mathbf{R}$ \\
\hline $9 \mathrm{R}-1,14$ & 68.64 & 328.9 & -22.4 & 44.0 & 326.2 & -22.3 & 14.0 & $\mathrm{R}$ \\
\hline $9 \mathrm{R}-2,14$ & 70.14 & 341.9 & -59.8 & 190.2 & 340.0 & -54.1 & 51.6 & $\mathrm{R}$ \\
\hline $9 \mathrm{R}-3,14$ & 71.64 & 59.7 & -38.7 & 83.0 & 68.3 & -34.8 & 18.5 & $\mathrm{R}$ \\
\hline $9 \mathrm{R}-4,14$ & 73.14 & 279.2 & -33.9 & 90.0 & 256.9 & -25.7 & 30.5 & $\mathbf{R}$ \\
\hline $9 \mathrm{R}-5,14$ & 74.64 & 177.6 & -25.3 & 80.2 & 155.6 & -51.0 & 16.8 & $\mathrm{R}$ \\
\hline $10 \mathrm{R}-1,53$ & 78.63 & 295.1 & 50.3 & 40.0 & 284.3 & 45.9 & 7.9 & $\mathrm{~N}$ \\
\hline $10 \mathrm{R}-2,30$ & 79.90 & 122.2 & 44.6 & 54.2 & 113.9 & 40.8 & 13.7 & $\mathrm{~N}$ \\
\hline IOR-3, 83 & 81.93 & 19.8 & 45.7 & 114.2 & 21.2 & 47.0 & 39.2 & $\mathrm{~N}$ \\
\hline $10 \mathrm{R}-4,13$ & 82.73 & 47.6 & 40.6 & 122.5 & 54.0 & 39.1 & 33.7 & $\mathrm{~N}$ \\
\hline $12 \mathrm{R}-1,12$ & 98.61 & 110.9 & -3.8 & 161.4 & 103.9 & -8.1 & 47.4 & $\mathrm{R}$ \\
\hline $12 \mathrm{R}-2,58$ & 99.48 & 268.9 & -18.6 & 99.3 & 270.2 & -22.7 & 31.3 & $\mathrm{R}$ \\
\hline $13 R-1,28$ & 107.38 & 52.2 & 3.2 & 26.2 & 42.3 & -37.0 & 25.9 & $\mathbf{R}$ \\
\hline 14R-1, 108 & 117.78 & 211.8 & -7.4 & 110.2 & 212.7 & -18.2 & 31.6 & $\mathbf{R}$ \\
\hline $14 \mathrm{R}-2,74$ & 118.94 & 344.2 & -3.9 & 113.2 & 43.4 & 1.9 & 38.3 & $?$ \\
\hline $14 \mathrm{R}-3,83$ & 120.53 & 66.3 & -27.3 & 142.1 & 68.2 & -29.5 & 43.2 & $\mathrm{R}$ \\
\hline $14 \mathrm{R}-4.21$ & 121.43 & 198.0 & -21.2 & 76.1 & 214.9 & -41.0 & 20.3 & $\mathrm{R}$ \\
\hline $14 \mathrm{R}-5,36$ & 123.06 & 131.6 & -23.8 & 142.4 & 128.3 & -28.7 & 40.0 & $\mathbf{R}$ \\
\hline $15 \mathrm{R}-1,123$ & 127.63 & 281.3 & -16.8 & 121.2 & 79.5 & -18.1 & 33.4 & $\mathbf{R}$ \\
\hline $15 \mathrm{R}-2,123$ & 129.13 & 239.3 & -31.3 & 161.1 & 243.8 & -34.6 & 46.4 & $\mathbf{R}$ \\
\hline $15 R-3,122$ & 130.63 & 95.5 & -23.5 & 128.7 & 101.6 & -26.9 & 35.0 & $\mathbf{R}$ \\
\hline $15 R-4,122$ & 132.13 & 343.3 & -30.1 & 105.3 & 343.1 & -32.8 & 26.2 & $\mathbf{R}$ \\
\hline $15 R-5,122$ & 133.63 & 125.2 & -34.8 & 170.7 & 124.1 & -39.1 & 51.9 & $\mathbf{R}$ \\
\hline $15 \mathrm{R}-6,122$ & 135.13 & 261.1 & -27.0 & 59.4 & 280.7 & -33.3 & 18.4 & $\mathbf{R}$ \\
\hline $15 \mathrm{R}-7,34$ & 135.74 & 273.1 & -25.9 & 71.9 & 273.8 & -30.0 & 21.0 & $\mathrm{R}$ \\
\hline $16 \mathrm{R}-1,109$ & 137.09 & 286.7 & 0.3 & 22.6 & 280.7 & 15.7 & 9.7 & $\mathrm{~N}$ \\
\hline $16 \mathrm{R}-2,109$ & 138.59 & 204.9 & 13.3 & 55.1 & 206.6 & 15.5 & 13.8 & $\mathrm{~N}$ \\
\hline 16R-3, 109 & 140.09 & 133.7 & 30.0 & 109.6 & 130.9 & 22.6 & 27.4 & $\mathrm{~N}$ \\
\hline $16 \mathrm{R}-4,109$ & 141.59 & 210.3 & 28.3 & 105.5 & 284.3 & 37.9 & 3.5 & $\mathrm{~N}$ \\
\hline $16 \mathrm{R}-5,109$ & 144.59 & 262.8 & 31.6 & 93.9 & 223.8 & 26.2 & 22.9 & $\mathrm{~N}$ \\
\hline 17R-1, 96 & 146.56 & 223.0 & -8.0 & 67.3 & 222.2 & -14.2 & 15.3 & $\mathrm{R}$ \\
\hline $17 \mathrm{R}-2,96$ & 148.06 & 197.9 & -40.1 & 37.7 & 220.0 & -54.3 & 10.0 & $\mathrm{R}$ \\
\hline $17 R-3,96$ & 149.56 & 188.9 & 50.1 & 10.0 & 201.7 & 61.7 & 2.8 & $\mathrm{~N}$ \\
\hline $17 R-4,96$ & 151.06 & 261.4 & 52.3 & 43.7 & 266.3 & 23.7 & 10.3 & $\mathrm{~N}$ \\
\hline $17 \mathrm{R}-5,91$ & 152.51 & 270.7 & -34.8 & 44.5 & 272.0 & -37.4 & 12.8 & $\mathbf{R}$ \\
\hline $18 \mathrm{R}-1,96$ & 156.26 & 331.1 & -7.6 & 265.3 & 327.2 & -6.5 & 70.3 & $?$ \\
\hline $18 R-2,96$ & 157.73 & 209.6 & 26.5 & 135.4 & 219.2 & 7.8 & 39.6 & $?$ \\
\hline $18 \mathrm{R}-3,115$ & 159.45 & 46.0 & 54.4 & 103.4 & 46.9 & 50.7 & 29.5 & $\mathrm{~N}$ \\
\hline $18 \mathrm{R}-4,108$ & 160.88 & 238.7 & -87.3 & 31.2 & 286.1 & -76.2 & 8.8 & $\mathrm{R}$ \\
\hline $18 \mathrm{R}-5,111$ & 162.41 & 170.1 & -16.0 & 43.7 & 160.0 & -21.6 & 10.4 & $\mathrm{R}$ \\
\hline 18R-6, 113 & 163.93 & 81.5 & -33.9 & 44.7 & 75.7 & -45.1 & 12.8 & $\mathrm{R}$ \\
\hline 19R-1, 48 & 165.48 & 169.7 & 15.2 & 83.9 & 151.8 & 52.7 & 13.8 & $\mathrm{~N}$ \\
\hline $20 \mathrm{R}-1,115$ & 175.85 & 262.3 & 1.5 & 9.6 & 218.8 & -6.4 & 3.2 & $\mathrm{R}$ \\
\hline $20 \mathrm{R}-2,86$ & 177.06 & 325.9 & 47.2 & 39.5 & 330.1 & 41.4 & 12.4 & $\mathrm{~N}$ \\
\hline $20 \mathrm{R}-3,57$ & 178.27 & 7.0 & 4.5 & 157.3 & 330.6 & 26.1 & 35.1 & $\mathrm{~N}$ \\
\hline $20 \mathrm{R}-4,146$ & 180.66 & 49.9 & 24.3 & 40.0 & 40.3 & 9.2 & 9.6 & $\mathrm{R}$ \\
\hline \multicolumn{9}{|l|}{$125-786 \mathrm{~A}-$} \\
\hline $1 \mathrm{H}-1,39$ & 0.39 & 157.5 & 9.8 & 31.7 & 156.2 & -45.5 & 8.7 & $\mathrm{R}$ \\
\hline $1 \mathrm{H}-2,39$ & 1.89 & 231.4 & -23.4 & 271.4 & 198.0 & -52.9 & 22.5 & $\mathrm{R}$ \\
\hline $1 \mathrm{H}-3,38$ & 3.38 & 324.2 & 11.2 & 193.6 & 180.0 & -48.0 & 15.2 & $\mathrm{R}$ \\
\hline $1 \mathrm{H}-4,43$ & 4.93 & 1.0 & 44.4 & 64.6 & 8.2 & 26.2 & 19.6 & $\mathrm{~N}$ \\
\hline IH-5, 43 & 6.43 & 345.6 & 45.3 & 167.2 & 13.1 & 35.1 & 27.3 & $\mathrm{~N}$ \\
\hline $1 \mathrm{H}-6,31$ & 7.86 & 16.7 & 34.7 & 41.9 & 21.1 & 13.3 & 5.9 & $?$ \\
\hline $1 \mathrm{H}-7,51$ & 9.51 & 172.8 & -20.9 & 29.7 & 173.8 & -34.5 & 13.6 & $\mathbf{R}$ \\
\hline $2 \mathrm{H}-1,134$ & 11.04 & 228.3 & -27.3 & 184.2 & 223.5 & 44.8 & 21.7 & $\mathrm{~N}$ \\
\hline
\end{tabular}


APPENDIX B (continued).

\begin{tabular}{|c|c|c|c|c|c|c|c|c|}
\hline \multirow[b]{2}{*}{$\begin{array}{l}\text { Core, section, } \\
\text { interval }(\mathrm{cm})\end{array}$} & \multirow[b]{2}{*}{$\begin{array}{l}\text { Depth } \\
\text { (mbsf) }\end{array}$} & \multicolumn{3}{|c|}{ Natural remanent magnetization } & \multicolumn{3}{|c|}{ Remagnetization at $40 \mathrm{mT}$} & \multirow[b]{2}{*}{ Polarity } \\
\hline & & $\begin{array}{l}\text { Declination } \\
\text { (degrees) }\end{array}$ & $\begin{array}{c}\text { Inclination } \\
\text { (degrees) }\end{array}$ & $\begin{array}{r}\text { Intensity } \\
(\mathrm{mA} / \mathrm{m})\end{array}$ & $\begin{array}{l}\text { Declination } \\
\text { (degrees) }\end{array}$ & $\begin{array}{l}\text { Inclination } \\
\text { (degrees) }\end{array}$ & $\begin{array}{l}\text { Intensity } \\
(\mathrm{mA} / \mathrm{m})\end{array}$ & \\
\hline $2 \mathrm{H}-2,13 \mathrm{I}$ & 12.51 & 24.8 & -46.2 & 147.9 & 347.5 & -46.3 & 19.3 & $\mathrm{R}$ \\
\hline $2 \mathrm{H}-3,132$ & 14.02 & 210.5 & 7.0 & 442.0 & 178.6 & 34.9 & 13.0 & $\mathrm{~N}$ \\
\hline $2 \mathrm{H}-4,133$ & 15.53 & 191.5 & 52.4 & 17.4 & 219.3 & 70.0 & 3.9 & $\mathrm{~N}$ \\
\hline $2 \mathrm{H}-5,137$ & 17.07 & 12.8 & -34.2 & 30.9 & 338.0 & -52.0 & 17.5 & $\mathrm{R}$ \\
\hline $2 \mathrm{H}-6,134$ & 18.54 & 193.9 & 43.1 & 67.9 & 201.7 & 40.9 & 19.2 & $\mathrm{~N}$ \\
\hline $2 \mathrm{H}-7,53$ & 19.23 & 198.4 & 51.8 & 68.8 & 200.9 & 60.6 & 15.7 & $\mathrm{~N}$ \\
\hline $3 \mathrm{H}-1,123$ & 20.43 & 110.9 & -69.9 & 39.6 & 198.5 & -61.1 & 16.2 & $\mathrm{R}$ \\
\hline $3 \mathrm{H}-2,123$ & 21.93 & 251.3 & -67.2 & 22.5 & 205.6 & -33.3 & 5.8 & $\mathrm{R}$ \\
\hline $3 \mathrm{H}-3,123$ & 23,43 & 47.4 & 31.5 & 36.6 & 19.4 & 3.4 & 2.8 & ${ }^{\mathrm{b}} \mathrm{R}$ \\
\hline $3 \mathrm{H}-4,123$ & 24.93 & 94.0 & -55.5 & 16.2 & 196.6 & -46.4 & 6.5 & R \\
\hline $3 \mathrm{H}-5,123$ & 26.43 & 39.9 & 37.0 & 25.6 & 9.7 & 48.3 & 4.8 & $\mathrm{~N}$ \\
\hline $3 \mathrm{H}-6,123$ & 27.93 & 225.0 & -46.5 & 21.9 & 227.9 & -57.8 & 12.1 & $\mathrm{R}$ \\
\hline $3 \mathrm{H}-7.36$ & 28.57 & 248.6 & -31.8 & 35.1 & 231.5 & -46.7 & 17.7 & $\mathrm{R}$ \\
\hline $4 X-1,117$ & 29.87 & 351.1 & 1.9 & 8.8 & 261.4 & 80.2 & 1.7 & $?$ \\
\hline $5 X-1,120$ & 39.40 & 22.2 & 53.2 & 23.6 & 21.4 & 62.0 & 6.6 & $\mathrm{~N}$ \\
\hline $5 X-2,120$ & 40.90 & 193.4 & 0.9 & 19.5 & 168.9 & 22.7 & 4.4 & $\mathrm{~N}$ \\
\hline $5 X-3,120$ & 42.40 & 50.9 & 35.5 & 43.5 & 6.9 & 60.1 & 9.7 & $\mathrm{~N}$ \\
\hline $5 X-4.72$ & 43.42 & 194.6 & 32.6 & 43.4 & 229.2 & 38.0 & 7.7 & $\mathrm{~N}$ \\
\hline $6 X-1,11$ & 48.75 & 228.3 & 44.5 & 54.2 & 228.9 & 36.0 & 13.4 & $\mathrm{~N}$ \\
\hline $6 \mathrm{X}-2,112$ & 50.22 & 31.3 & 76.4 & 63.7 & 63.7 & 27.6 & 16.5 & $\mathrm{~N}$ \\
\hline $6 X-3,112$ & 51.52 & 37.6 & 21.2 & 19.3 & 321.4 & -8.6 & 2.8 & $\mathrm{R}$ \\
\hline $6 X-4,112$ & 53.22 & 38.9 & -18.5 & 35.9 & 30.0 & -46.7 & 13.0 & $\mathrm{R}$ \\
\hline
\end{tabular}

${ }^{a} \mathrm{~N}$, normal; R, reversed; ?, indeterminate.

${ }^{\mathrm{b}}$ Remanent magnetization at $50 \mathrm{mT}$. 